\title{
Multicolor photometry and spectroscopy of the yellow supergiant with dust envelope HD 179821=V1427 Aquilae
}

\author{
N.P. Ikonnikova* O.G. Taranova, V.P. Arkhipova, \\ G.V. Komissarova, V.I. Shenavrin, V.F. Esipov, M.A. Burlak, V.G. Metlov \\ Sternberg Astronomical Institute, \\ Moscow State University (SAI MSU), Universitetskii pr. 13, Moscow, 119992 Russia
}

\begin{abstract}
We present the results of multicolor ( $U B V J H K L M)$ photometry (2009-2017) and low-resolution spectroscopy (2016-2017) of the semi-regular variable V1427 Aql=HD 179821, a yellow supergiant with gas-dust envelope. The star displays low-amplitude $\left(\Delta V<0 .^{m} 2\right)$ semi-periodic brightness variation superimposed on a long-term trend. The light curve shape and timescale change from cycle to cycle. There are temperature variations characteristic for pulsations, and brightness oscillations with no significant change of color are also observed. The $U B V$ data for the 2009-2011 interval are well reproduced by a superposition of two periodic components with $P=170^{d}$ and $P=141^{d}$ (or $P=217^{d}$ - the one year alias of $P=141^{d}$ ). The variation became less regular after 2011, the timescale increased and exceeded 250 . An usual photometric behavior was seen in 2015 when the star brightness increased by $0 .{ }^{m} 25$ in the $V$ filter in 130 days and reached the maximum value ever observed in the course of our monitoring since 1990. In 2009-2016 the annual average brightness monotonically increased in $V, J, K$, whereas it decreased in $U$ and $B$. The annual average $U-B, B-V$, and $J-K$ colors grew, the star was getting redder. The cooling and expanding of the star photosphere along with the increasing of luminosity may explain the long-term trend in brightness and colors. Based on our photometric data we suppose that the photosphere temperature decreased by $\sim 400 \mathrm{~K}$ in the 2008-2016 interval, the radius increased by $\sim 24 \%$, and the luminosity grew by $\sim 19 \%$. We review the change of annual average photometric data for almost 30 years of observations. Low-resolution spectra in the $\lambda 4000-9000 \AA$ wavelength range obtained in 2016-2017 indicate significant changes in the spectrum of V1427 Aql as compared with the 1994-2008 interval, i.e. the BaII and near-infrared CaII triplet absorptions have turned stronger while the OI $\lambda 7771-4$ triplet blend has weakened that points out the decrease of temperature in the region where the absorptions are formed. The evolutionary stage of the star is discussed. We also compare V1427 Aql with post-AGB stars and yellow hypergiants.
\end{abstract}

Keywords: post-AGB, post-RSG, yellow hypergiants, spectral and photometric observations, brightness variability, evolution

\footnotetext{
*E-mail: ikonnikova@gmail.com
} 


\section{INTRODUCTION}

The evolutionary status of the yellow supergiant with infrared (IR) excess HD 179821 (BD-00 3679 $=\mathrm{SAO} 124414=$ IRAS $19114+0002=\mathrm{V} 1427 \mathrm{Aql})$ is not entirely clear. No doubt it is an evolved star as implied by the presence of a spherical gas-dust circumstellar envelope formed through mass loss episodes during earlier stages of evolution. But the initial mass of HD 179821 is still under the question. Some data point to intermediate mass $\left(M_{Z A M S}=1 \div 8 M_{\odot}\right)$, and then HD 179821 is a post-AGB supergiant and a planetary nebula progenitor (Pottasch and Parthasarathy 1989; Hrivnak et al. 1989; Ferguson and Ueta 2010); other data favor the idea that HD 179821 is a massive star $\left(M_{Z A M S}>\right.$ $20 M_{\odot}$ ) which has evolved off the Red Supergiant (RSG) and later can undergo a Supernova II outburst, lose its envelope, and become a neutron star (Jura et al. 2001; Oudmaijer et al. 2009; Şahin et al. 2016). Both hypotheses were discussed in detail in Reddy and Hrivnak (1999), Josselin and Lebre (2001), Coroller et al. (2003), Arkhipova et al. (2009).

Photometric instability is an important feature of HD 179821. We discovered its variability on the basis of our photometric observations in 1990-1992 (Arkhipova et al. 1993). In the General Catalogue of Variable Stars, the object was designated as V1427 Aql and associated with semi-regular SRd-type variables (Samus 2017). Further investigations carried out in 1993-1999 (Arkhipova et al. 2001), in 1994-2000 (Hrivnak 2001), in 1999-2000 (Coroller et al. 2003), and in 2000-2008 (Arkhipova et al.2009) revealed low-amplitude $\left(\Delta V=0 \cdot^{m} 1-0 \cdot^{m} 2\right)$ brightness oscillations with cycle lengths of nearly $200 \mathrm{~d}$ and also a general change in brightness and colors. The stars at the late stages of evolution are known to experience some instability which differs for post-AGB supergiants and yellow hypergiants, the latter having evolved off the RSG. Therefore knowing photometric variability peculiarities is of particular importance for stars of uncertain evolutionary status.

In this work, we present new multicolor photometric data for V1427 Aql obtained in 2009-2017 and analyze the overall change in brightness and colors over the whole period of our photometric observations since 1990. We also adduce low-resolution spectral observations obtained in 2016-2017 and compare them with earlier data.

\section{OBSERVATIONS}

\section{$U B V$-photometry}

We have been carrying out photometric observations of V1427 Aql since 1990. The results of $U B V$ light curve study over the 1990-2008 interval were reported in Arkhipova et al. (1993, 2001, 2009).

After 2008 we continued to monitor V1427 Aql at the Crimean SAI MSU Station with the 60-cm Zeiss reflector equipped with the photon counting $U B V$-photometer (Lyutyi 1971). The diameter of the chosen aperture was $27^{\prime \prime}$. The star SAO 124412 ( $\mathrm{sp}=\mathrm{G} 8 \mathrm{IV}$ ) with $U=9 \cdot^{m} 87, B=9{ }^{m} 56, V=8 .^{m} 77$ was used as a comparison star. We estimate the average uncertainty to be about $0 .^{m} 01$. Table 1 presents $U B V$-magnitudes for V1427 Aql in 2009-2017.

\section{IR-photometry}

IR-photometric observations were made at the Crimean SAI MSU Station with the 125-cm telescope. The photometer with a liquid nitrogen cooled photovoltaic indium antimonide (InSn) detector (Shenavrin et al. 2011) was installed at the Cassegrain focus, the output aperture was $\sim 12^{\prime \prime}$. The star BS $7377(\mathrm{Sp}=\mathrm{F} 0 \mathrm{IV})$ was used as a photometric standard, and its magnitudes were taken from the catalog of Johnson et al. (1966): $J=2 \cdot{ }^{m} 75, H=2 \cdot{ }^{m} 61, K=2 \cdot{ }^{m} 57, L=2 \cdot{ }^{m} 54, M=2 \cdot{ }^{m} 59$. Our photometric errors did not exceed $0 .{ }^{m} 05$ for the $M$-band and $0 .{ }^{m} 02$ for the others. In an earlier paper (Arkhipova et al. 2009), we review the IR photometry made in 1992-2000 and in 2008 (two nights) and 
also the data obtained by other researchers before 1992. Table 2 present new JHKLM-magnitudes for HD 179821 in 2009-2017.

\section{Spectral observations}

We have been making low-resolution spectral observations of V1427 Aql since 1994. The review of spectral data obtained in 1994-2008 can be found in Arkhipova et al. (2009). In this work, we present the recent observations made in 2016-2017. Spectra with a wavelength coverage of $\lambda 4000-9000 \AA$ were obtained at the Crimean SAI MSU Station with the 125-cm reflector and the fast A-spectrograph. An SBIG ST-402 CCD detector was employed providing a spectral resolution of $\sim 2.2 \AA$ per pixel.

The log of observations is presented in Table 3, where we list the observing date and the standard star used for calibration. On October 25, 2016, the standard star was not observed because the weather got worse.

The spectra were processed with the use of the CCDOPS standard program and the SPE program (Sergeev and Heisberger 1993). The data were flux-calibrated using spectrophotometric standard stars, the spectral energy distribution for them in the $\lambda 4000-7650 \AA$ wavelength range was taken from the spectrophotometric catalog of Glushneva et al. (1998) and extended to $\lambda 9000 \AA$ according to the stellar spectral flux library of Pickles (1998).

\section{ANALYSIS OF PHOTOMETRIC AND SPECTRAL VARIABILITY OF V1427 AQL}

\section{Photometric variability of V1427 Aql in 2009-2017.}

In Fig. 1 we present the $U B V$ light and $U-B, B-V, U-V$ color curves for V1427 Aql observed in Crimea in 2009-2017. During that time the object's behavior was not strictly homogeneous.

In 2009-2010 ( $\mathrm{JD}=24$ 54970-55516) V1427 Aql displayed a regular cyclical variation in brightness. Colors varied strictly in phase with brightness: the object was bluer when brighter and redder when fainter. In 2010, when the pulsation pattern appeared the most distinct, the full range in brightness was of $\Delta V=0 .^{m} 18$ and the $B-V$ color varied over a range of $0 \cdot^{m} 58-0 .^{m} 66$.

A smaller brightness range was present in the 2011 observations with a peak-to-peak variation of nearly $0 .^{m} 1$ in $U B V$. Brightness variations were not accompanied by significant change in color.

Brightness variations turned to be less regular in 2012-2014 (JD=24 56040-56605) with maximum $V$ amplitude of $0 .^{m} 15$, the timescale of brightness variability increased and became comparable to the duration of an observational season.

In 2015 we performed $U B V$-observations of V1427 Aql during $138 \mathrm{~d}$, from May 19 till October $3(\mathrm{JD}=2457162-57299)$. In the course of this time, the star brightened quickly by $\Delta V \sim 0{ }^{m} 25$, $\Delta B \sim 0 .^{m} 33$ and $\Delta U \sim 0 .^{m} 40$. By $\mathrm{JD}=2457299$ the $V$ brightness reached the value of $7 .^{m} 85$ that appeared the maximum brightness since 1990, over the whole interval we had observed V1427 Aql. Unfortunately, the estimate obtained that night was the last in 2015 , so we could not fix the moment of maximum in that cycle. The evolution of the stellar colors was unusual: the brightness increasing from $V=8 \cdot{ }^{m} 05$ to $V=7 .^{m} 91$, the $B-V$ and $U-B$ colors hardly varied indicating that the luminosity growth was due to the increase in the star radius but not to the rise of the temperature; then the star became a little bluer.

The next observational season for V1427 Aql started on April 7, 2016 (JD=24 57486) and lasted for 228 days and during this time the stellar brightness increased systematically in all bands displaying local extremes in the $B$ and $U$ light curves: minimum brightness at JD=24 57517 and maximum 

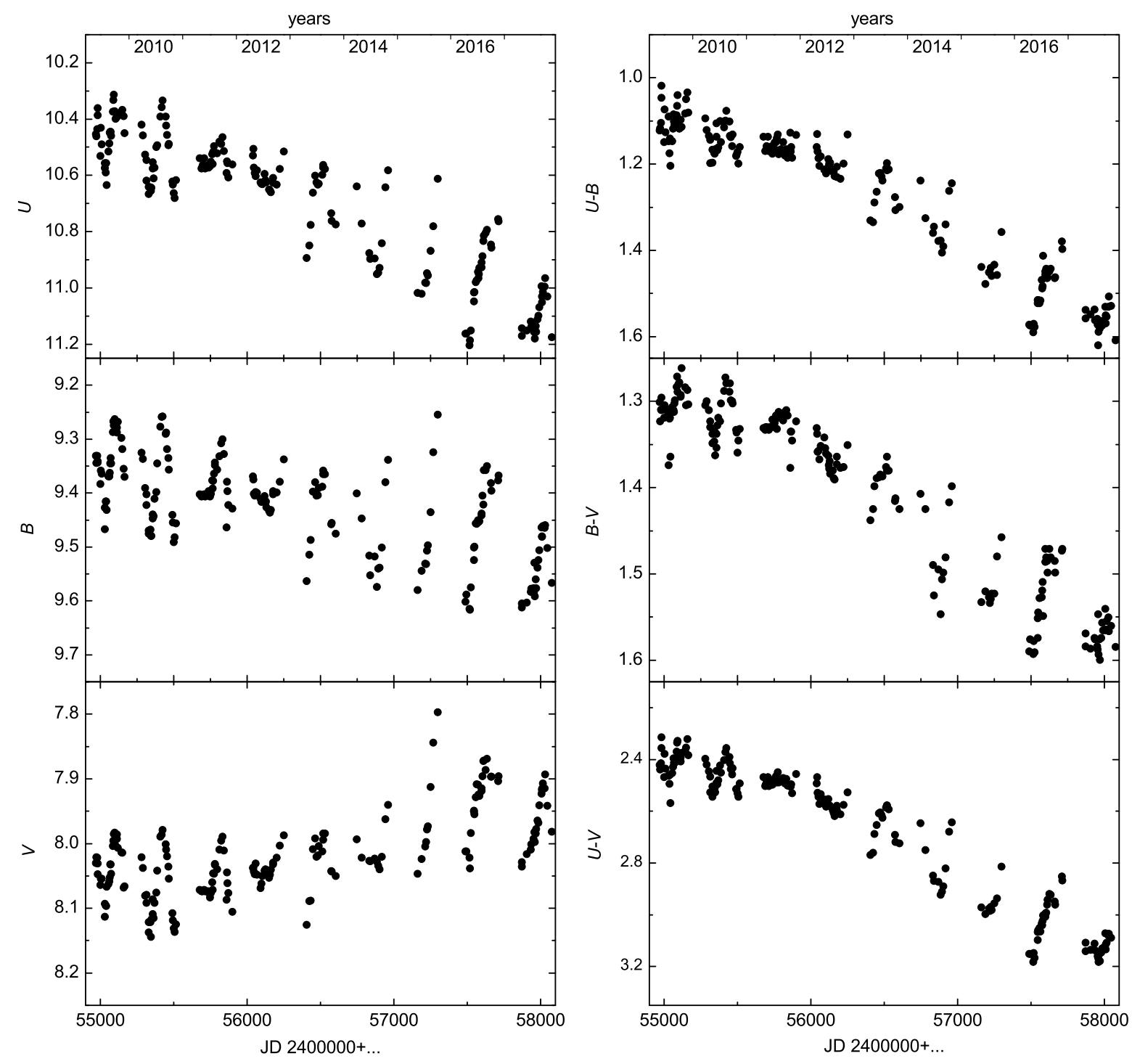

Figure 1: Light and color curves of V1427 Aql in 2009-2017. 

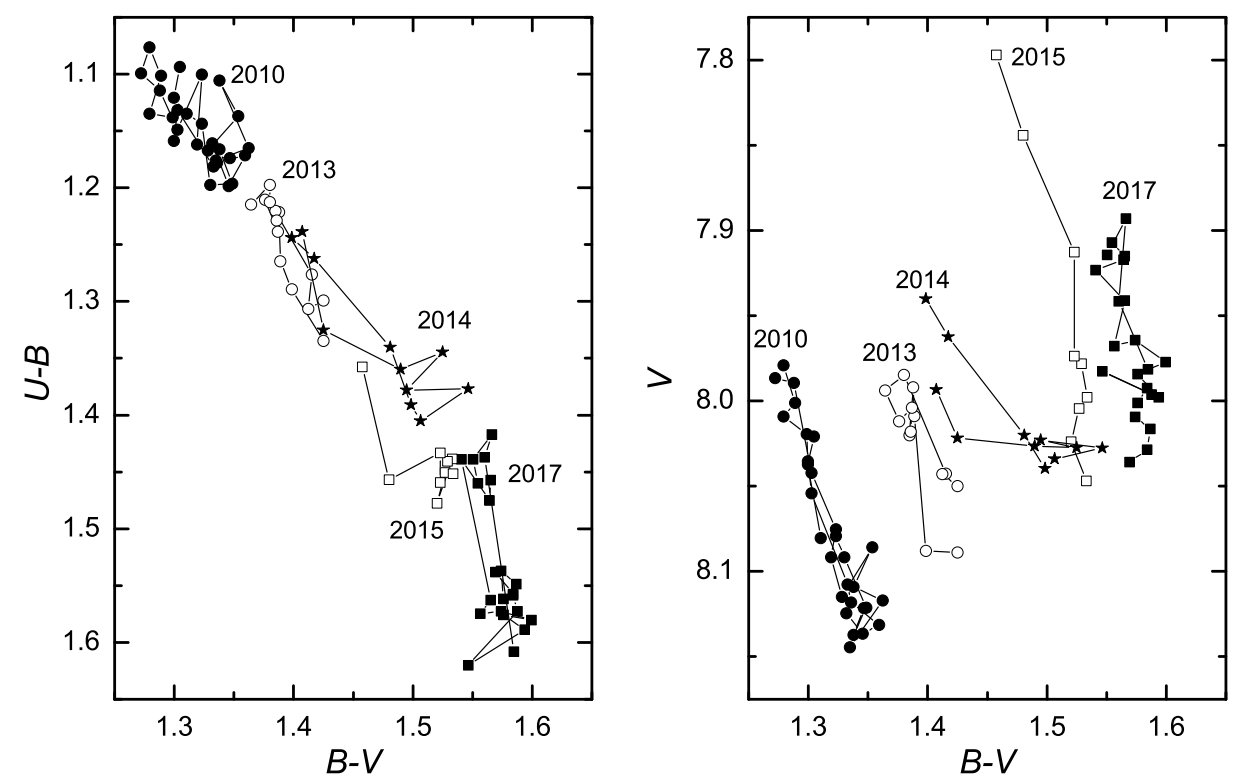

Figure 2: Color-color and color-magnitude diagrams for V1427 Aql covering five observing seasons. Numbers indicate years.

brightness at JD 24 57630. The star brightened by $\Delta V \sim 0 .^{m} 15, \Delta B \sim 0 .{ }^{m} 27, \Delta U \sim 0 .^{m} 39$ having turned bluer by $\Delta(B-V) \sim 0 .^{m} 10$ and $\Delta(U-B) \sim 0 .^{m} 15$.

The stellar brightness was increasing for 146 days in 2017, beginning April 26 (JD=24 57870), reached its maximum near September $18(\mathrm{JD}=2458015)$, and then the star started getting fainter. The amplitude of light variation was up to $0 \cdot^{m} 13$.

Fig. 2 plots the color-color and the color-magnitude diagrams to demonstrate the difference in color-magnitude relation for various observational seasons.

Apart from regular oscillations of brightness and color which are more or less regular and correspond to pulsations and maybe some other type of atmospheric instability the star displayed a general trend in brightness and color over the observing interval: on average the $V$ brightness increased from season to season with the $B$ and $U$ brightness getting fainter and the $U-B$ and $B-V$ colors getting redder.

In the near-IR range, the $J(1.25 \mu \mathrm{m})$ and $K(2.2 \mu \mathrm{m})$ observations are the most numerous for the 2009-2017 interval. Fig. 3 shows $J, K, L$ and $M$ light and $J-K, H-K, L-M$ color curves. Stellar pulsations are responsible for brightness variations within each season with the amplitude not exceeding $\sim 0 .^{m} 1$ and being largest in the $J$ band. The $J H K L$ brightness appeared to increase systematically from season to season in 2009-2015. By 2015 the star showed an increase in brightness of $0 .^{m} 4$ in $J$ while colors turned redder, for example, the $J-K$ color increased by $\sim 0 .^{m} 1$. It's worth mentioning that a significant excess of $M$ light was observed in 2015.

In the section "Secular trend in brightness and color" we discuss the gradual change of seasonal average photometric parameters of the star. 

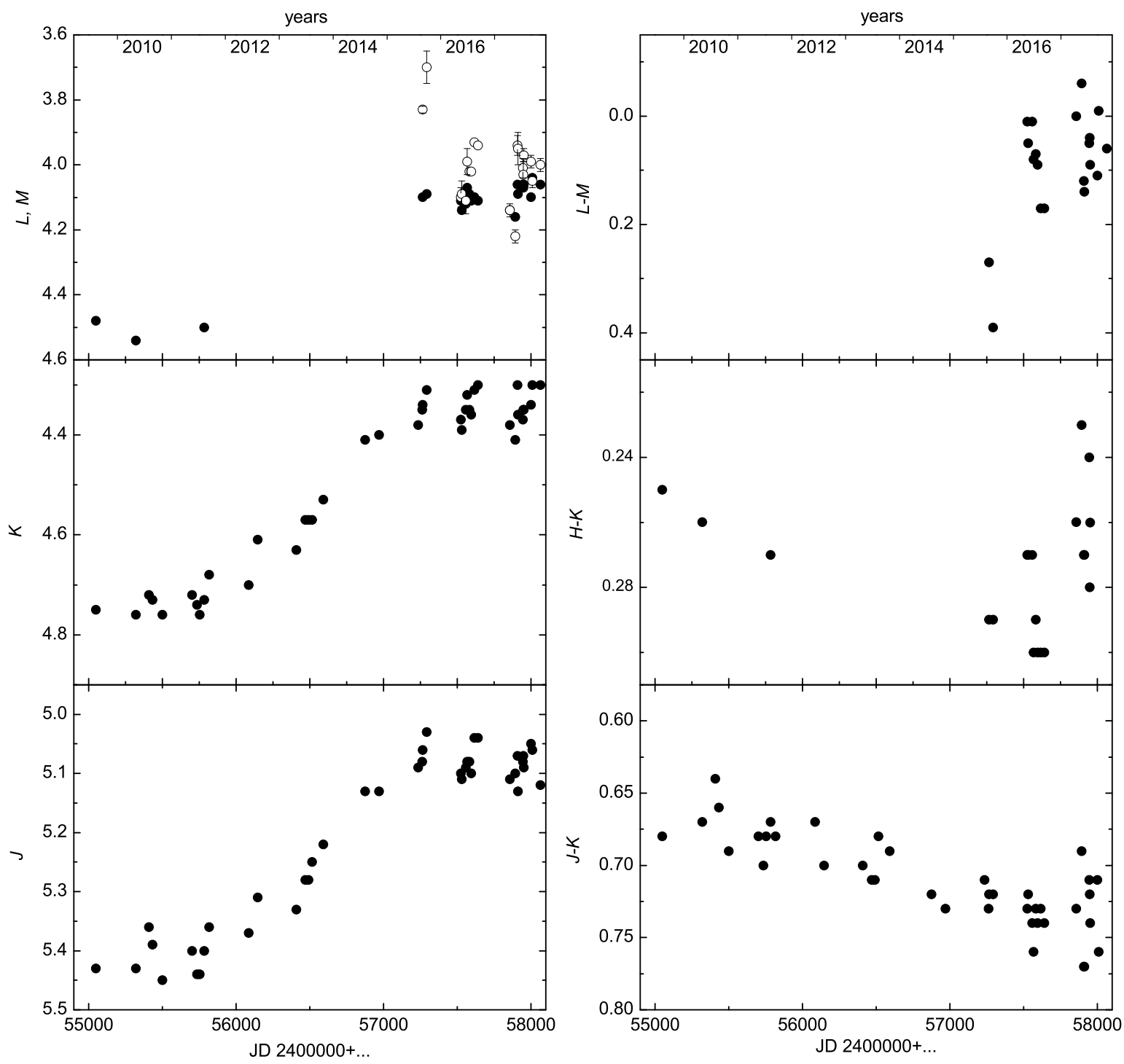

Figure 3: Light and color curves of V1427 Aql in near-IR range in 2009-2017. In the upper left panel points represent $L$ magnitudes, open circles $-M$ magnitudes. 


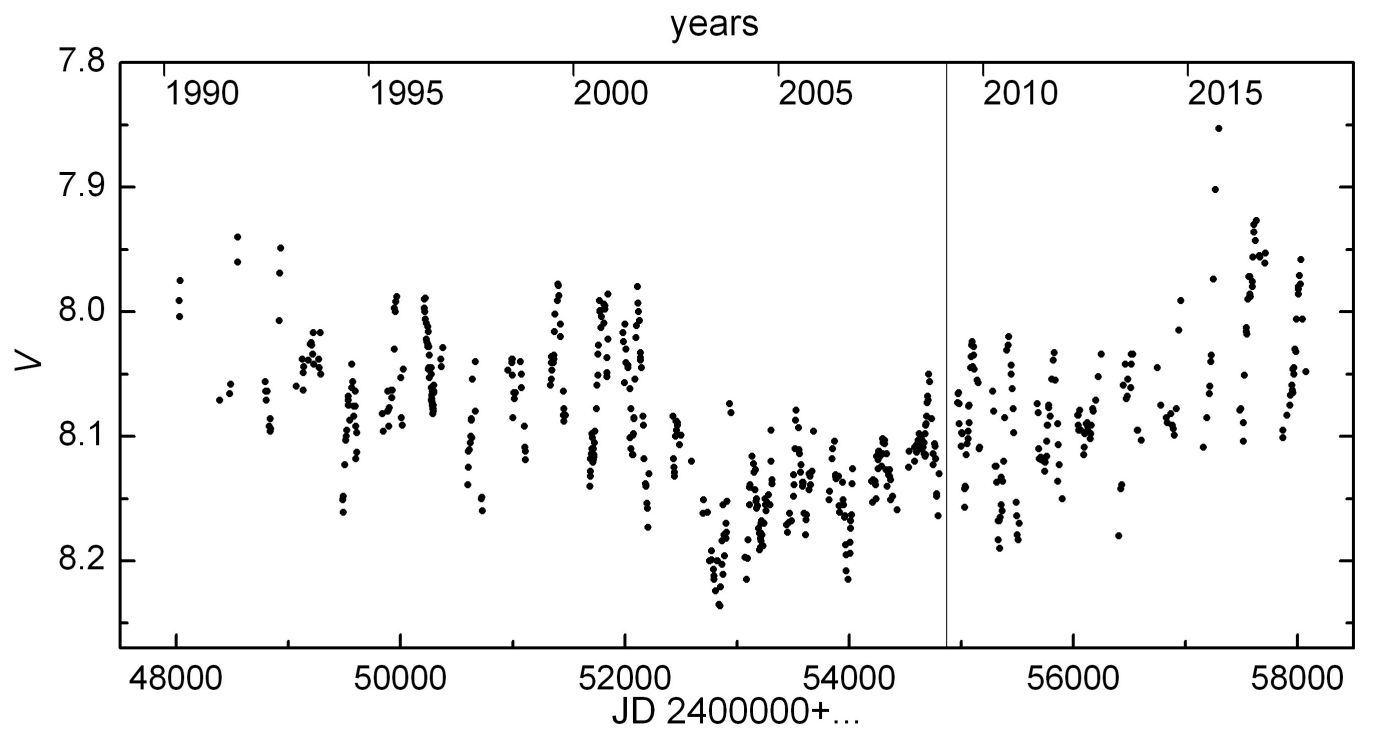

Figure 4: $V$-band light curve for V1427 Aql observed in 1990-2017. More recent data obtained in 2009-2017 are located to the right from the vertical line.

\section{Period analysis}

Fig. 4 shows the entire $V$ light curve observed in 1990-2017. As one can see the star displays semiregular brightness variation with changing amplitude that is typical for oscillations with multiple periods.

Search for periodicity for V1427 Aql was carried out by Arkhipova et al. (2001, 2009) on the basis of photometric data obtained in 1990-1999 and in 2000-2008 respectively and also by Coroller et al (2003) on the basis of their own observations made in 1999 and 2000 with the data of Arkhipova et al. (2001) and Hrivnak (2001) engaged.

Using $U B V$ observations of V1427 Aql made in 1994-1999 (Arkhipova et al. 2001), we found two periods of $P_{0}=205^{d}$ and $P_{1}=142^{d}$ and suggested that they correspond to the fundamental mode and the first overtone of pulsation. Coroller et al. (2003) got similar values.

Arkhipova et al. (2009) pointed out a possible change of pulsation period and phase for V1427 Aql after the year 2000. We found two periods $P_{0}=196 \pm 5^{d}$ and $P_{1}=128 \pm 5^{d}$ for the interval 2001-2008. These periods are the one year alias of each other. It turned difficult to determine which of them is a true period of pulsations and which is a spurious one. The observations made in 2001, 2004 and 2005 , when the pulsation pattern was distinct, agree better with $P_{1}=128 \pm 5^{d}$ while the timescale of brightness change was closer to $200^{d}$ in 2002, 2003, 2006 and 2007. 
In this work, we present frequency analysis basing on the new $U B V$-data acquired in 2009-2017.

To search for periods, we used the program PERIOD04 (Lenz and Breger 2005) and also the program EFFECT created by V.P. Goranskij which applies a Fourier transform for arbitrary data spacing (Deeming 1975).

The frequency spectrum derived from the $U B V$ data for the 2009-2011 interval shows a clear peak at $P_{1}=170 \pm 5^{d}$, and with this peak removed, two other periods dominate the residual spectrum: $P_{2}=141 \pm 5^{d}$ and $P_{3}=217 \pm 5^{d}$. The frequency spectrum that covers the period range of $10^{d}-400^{d}$ and includes the 2009-2011 $B$ data is shown in Fig. 5. Ten estimates of $J$ brightness obtained in the interval 2009-2011 yield two periods of $137^{d}$ and $216^{d}$ which are the one year alias of each other and are close to the values $P_{2}$ and $P_{3}$ derived from the $U B V$ data.

In 2012-2017 the period increased and exceeded observing intervals. The timescale of brightness variability rose up to $260^{d}$ in 2012-2014. The frequency analysis reveals a period of nearly $370^{d}$ for the 2015-2017 subset of $V$ data. 22 estimates in the $J$ band made in 2015-2017 yield a period of $175^{d}$ but its significance value is not high.

The nature of V1427 Aql semi-periodic variability is not entirely clear. Along with the cyclic change in brightness which is similar to pulsation, variations of another type are present perhaps being a result of variable stellar wind.

\section{Secular trend in brightness and color}

Long-term monitoring of V1427 Aql from 1990 till 2017 leads to the detection of a secular trend in brightness and color. In Fig. 6, which shows the $V$ light and $U-B, B-V$ color curves for the whole observing interval including data from Arkhipova et al. (2009) and also the change of $J$ magnitude and $J-K, K-L$ colors from 1987 till 2017 including previously published (Hrivnak et al.1989; Kastner and Weintraub 1995; Arkhipova et al. 2009) and new data, each point is an annual mean value.

The $U-B$ and $B-V$ colors were turning monotonically bluer from 1990 to 1994 with the $V$ brightness decreasing. The $B-V$ color remained quite constant after 1994, and the $U-B$ color continued to decline. The 1998-2000 data reveal a tendency for the object to get redder, then the star turned bluer again in 2001-2002, and in 2002 the $U-B$ and $B-V$ colors reached the values that appeared minimum for the whole observing interval. Later, after the year 2002, the object was getting redder brightening in the $V$ band. By 2016 the $V$ brightness reached the 1990 value with the colors being redder than when our observations started.

Hrivnak et al. (1989) and Kastner and Weintraub (1995) were the first to obtain near-IR observations of V1427 Aql in 1987-1988. By 1992 when we started monitoring V1427 Aql in the near-IR, the star was found $\sim 0 .^{m} 3$ fainter in $J$, and the $J-K$ color turned out bluer. From 1992 till 2000 the $J$ brightness was changing by not more than $0 .{ }^{m} 1$ with the $J-K$ color variation being within the range of $0 .{ }^{m} 05$. From 2001 till 2007 the star was not observed in the near-IR. Since 2008 the star was getting brighter in the $J, H, K$ and $L$ bands with the $J-H$ and $J-K$ colors increasing, while from 2015 there appeared a tendency for the star to get fainter in all filters and for the $J-H$ and $J-K$ colors to decrease. In the 1-5 $\mu \mathrm{m}$ wavelength range no IR-excess was detected for the star, so the energy emitted in this range is attributed entirely to the stellar photosphere and determined by its parameters.

Fig. 7 shows the color-color diagram for V1427 Aql with the annual mean $U B V$ magnitudes used for the interval 1990-2017. As is apparent from the figure the star was getting bluer in 1990-2002, and then a systematic reddening began. The fastest change in color occurred recently in 2013-2017. During this interval, the seasonal mean colors increased significantly, by $\Delta(U-B) \sim 0 .^{m} 3$ and $\Delta(B-V) \sim 0 .{ }^{m} 2$. As we reported in Arkhipova et al. (2009) the long-term trend in colors coincides 


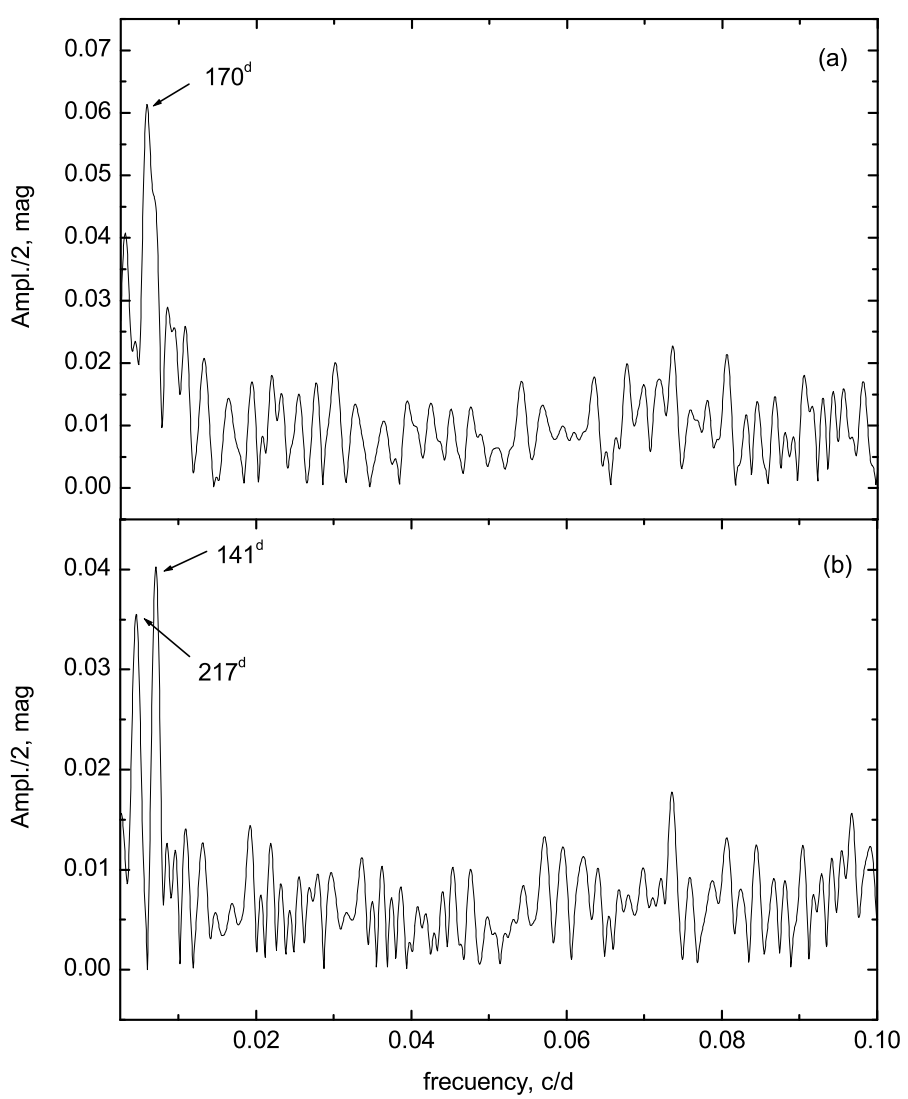

Figure 5: The frequency spectrum based on the $B$ data obtained in 2009-2011 for V1427 Aql: a) the initial spectrum including the full set of observations; b) the residual spectrum with the primary period component removed from the light curve. 

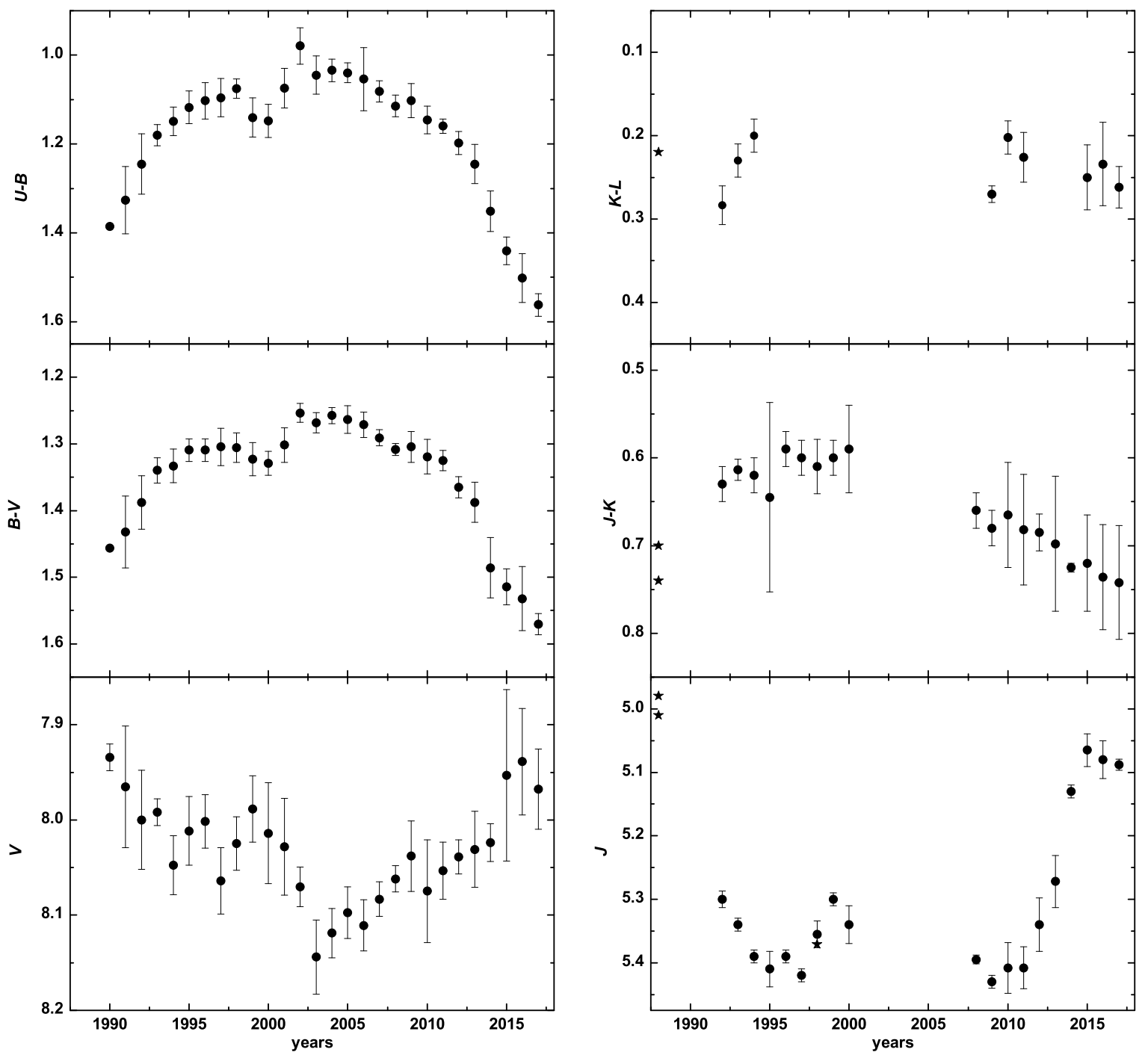

Figure 6: Optical and near-IR light and color curves for V1427 Aql composed of annual mean values. Points represent our data, asterisks - original estimates from Hrivnak et al. (1989), Kastner and Weintraub (1995) and 2MASS catalog. Vertical bars correspond to the amplitude of variation within a season. 


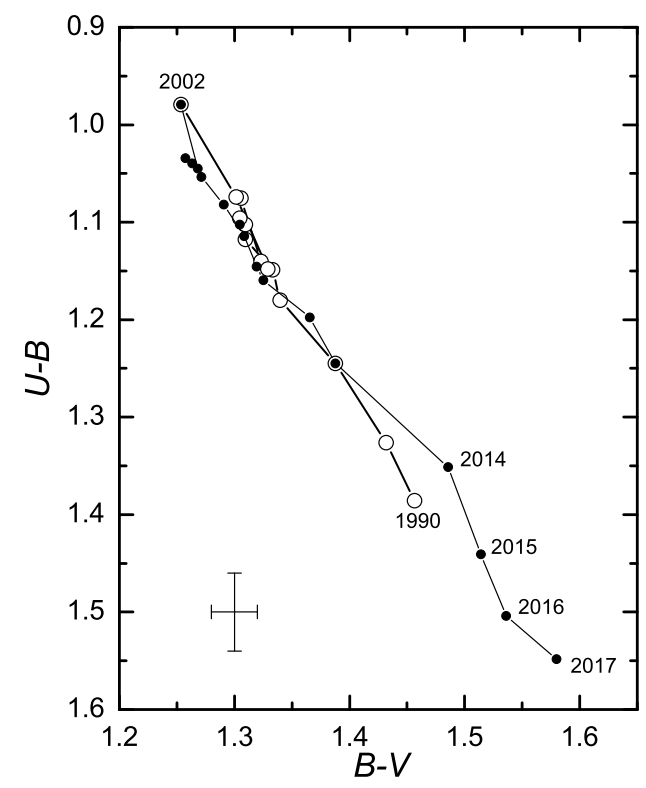

Figure 7: $(B-V)-(U-B)$ color-color diagram for the annual mean magnitudes of V1427 Aql. Open circles represent the 1990-2002 data; points correspond to the 2002-2017 interval. The numbers indicate years.

with the $B-V$ and $U-B$ colors varying in case of pulsations. That is why we consider the main cause of the long-term trend in colors to be the change of the stellar temperature: growth from 1990 till 2002 and then systematic decline towards 2017.

Fig. 8 shows the color-magnitude relations based on the seasonal average optical and IR magnitudes for V1427 Aql. The star moves similarly in both diagrams. After the first observations of V1427 Aql in 1987-1988, the star appeared to show a monotonic decrease in the $V$ and $J$ brightness along with getting bluer that has turned to brightening and reddening in recent years. The long-term trend in brightness and colors reflects the change in temperature and luminosity of the star photosphere. In Fig. 8 the reddening trajectory for the total and selective interstellar extinction laws $A_{V}=3.1 \times$ $E(B-V)$ and $A_{J}=1.50 \times E(J-K)$ adopted from Koornneef (1983) is shown with arrows. If the circumstellar extinction changed, its range was not larger than $\Delta E(B-V)=0 .{ }^{m} 02$.

\section{Spectral variability of V1427 Aql in 1994-2017.}

Our spectral observations of V1427 Aql obtained in 1994-2008 were discussed in Arkhipova et al. (2009). In that work, we estimated the temperature and spectral class of the star having compared it with other objects, and also reported about the $\mathrm{H} \alpha$ line variability. We did not find other changes in spectrum during the mentioned interval.

We did not obtain spectra for the star from 2009 till 2015, recommenced observing V1427 Aql in May 2016 and continued until October 2017 (see Table 3).

Analysing new observational data we found out that the spectrum of V1427 Aql had changed a lot between the 1994-2008 interval and the year 2016. Fig. 9 shows the continuum normalized spectra for V1427 Aql in the $\lambda 4200-9000 \AA$ wavelength range obtained on June 7, 2008, and July 27, 2016. 

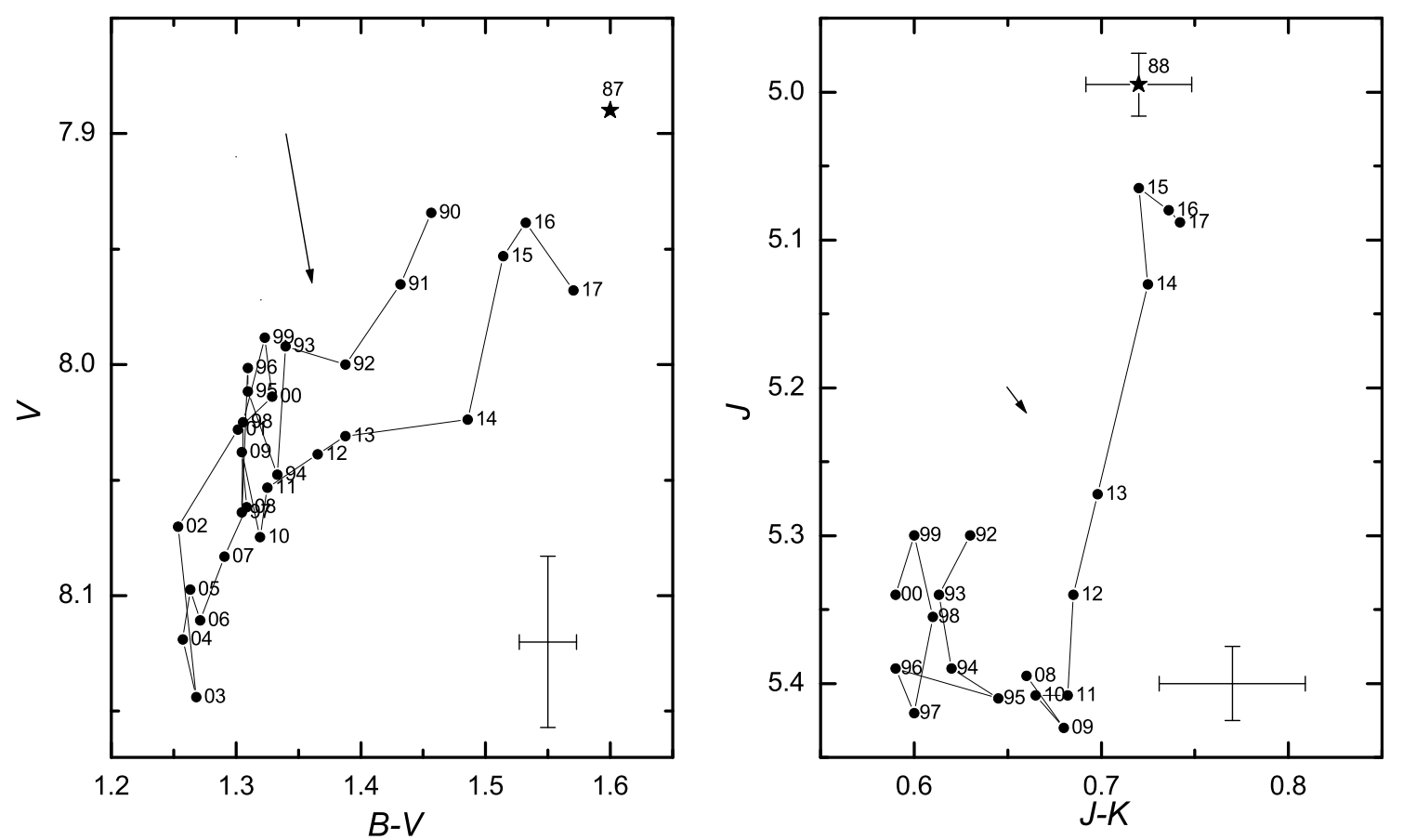

Figure 8: The $(B-V)-V$ and $(J-K)-J$ color-magnitude diagrams. The points represent the seasonal means of our data, the asterisks - the observations from Hrivnak et al. (1989). The numbers indicate years. The reddening trajectory for the standard interstellar extinction law is shown with arrows. The arrows' length corresponds to the extinction associated with $E(B-V)=0 .{ }^{m} 02$. 


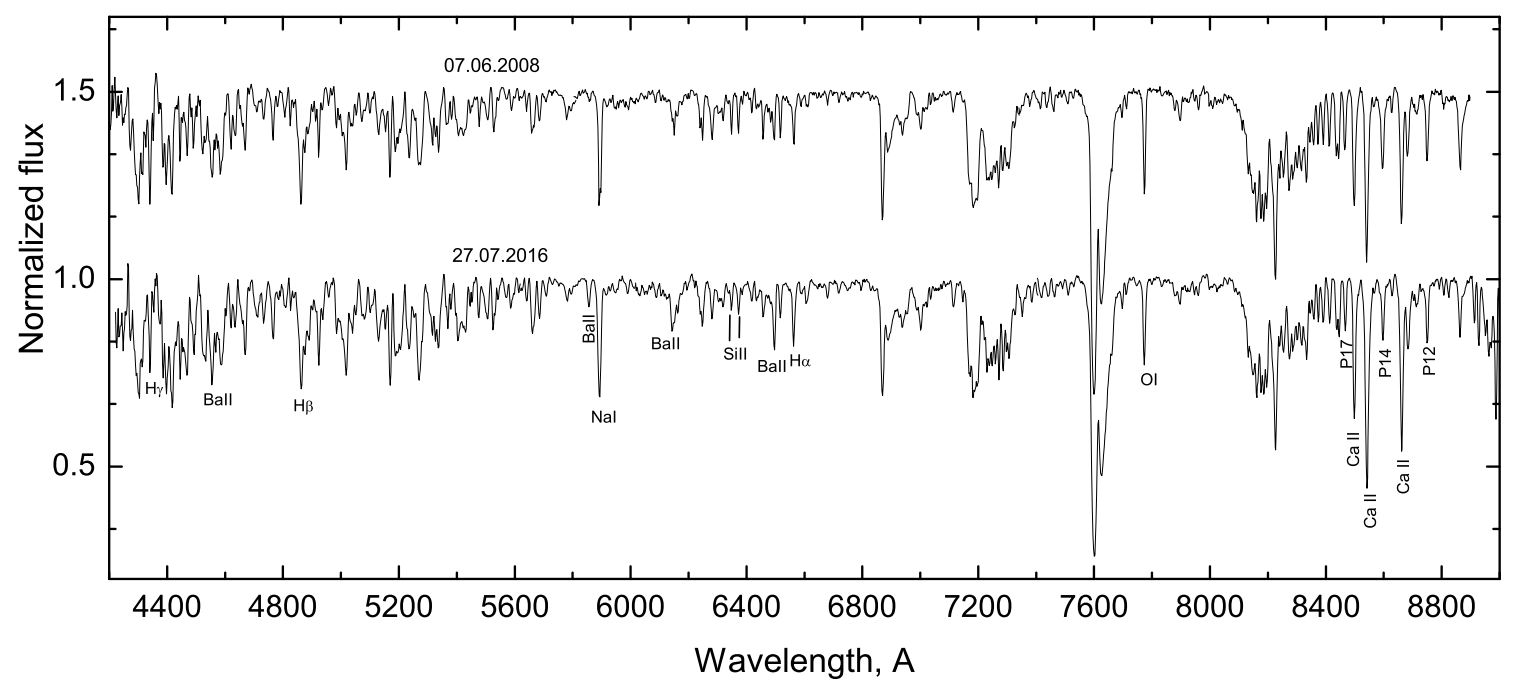

Figure 9: The continuum normalized spectra for V1427 Aql obtained on June 7, 2008, and on July 27,2016 . The upper spectrum is plotted with a vertical shift of 0.5 .

The spectral lines to look at are marked in the plot. The BaII lines are seen to have got deeper from 2008 to 2016, the absorptions related to the CaII IR-triplet strengthened. Besides the OI triplet at $\lambda 7771-4 \AA$ and Paschen lines weakened, the G-band to $\mathrm{H} \gamma$ line relation changed.

For the quantitative analysis, we measured the equivalent widths $E W$ of several absorptions, namely $\mathrm{H} \alpha$, BaII $\lambda 5857,6497$, FeII $\lambda 6516$, the OI $\lambda 7771-4$ triplet, P12, P14, and P17 hydrogen lines, the CaII triplet $(\mathrm{CaT}=\lambda 8498+\lambda 8542+\lambda 8662)$, using the spectra from Arkhipova et al. (2009) and those obtained in 2016 and 2017 and here present them in Table 4. It is worth mentioning that due to the given spectral resolution the CaII triplet components were severely blended with the P13, P15 and P16 hydrogen lines. The estimated error was about $10 \%$.

Fig. 10 shows the evolution of the equivalent widths of several lines.

In our spectra, the $\mathrm{H} \alpha$ equivalent width varies more than twice from $0.7 \AA$ to $1.97 \AA$. Highresolution spectra demonstrate that $\mathrm{H} \alpha$ has a complicated profile with varying emission components on the blue and red sides and with the absorption feature undergoing significant changes in width and radial velocity (Tamura and Takeuti 1991; Zacs et al. 1996; Kipper 2008; Şahin et al. 2016). As stated in Klochkova (2014), mass loss rate, stellar wind velocity, kinematics and optical depth of the circumstellar matter are the principal factors that determine the shape and variability of the $\mathrm{H} \alpha$ profile for the far evolved stars surrounded by spatially extended envelopes.

We can not measure the OI $\lambda 7771-4$ triplet components separately. The OI blend equivalent width was estimated to be $2.9 \pm 0.1 \AA$ in 1994-1999 (an average value), $2.57 \pm 0.30 \AA$ in 2008 , and by 2016 it decreased to $2.33 \pm 0.10 \AA$. In literature we could find few $E W(\mathrm{OI})$ estimates: $2.6 \AA$ (Reddy and Hrivnak 1999), $2.9 \AA$ (Pooley 2003), $3.0 \AA$ (Sánchez Contreras et al. 2008) - derived from highresolution spectra obtained on October 15-16,1997, May 7, 1999, and June 2, 2003, respectively.

The oxygen triplet is a good indicator of luminosity for A-G supergiants. As has been pointed out repeatedly, V1427 Aql has an extremely high $E W(\mathrm{OI})$ value that leads to the absolute magnitude estimate of $M_{V} \approx-8 \cdot{ }^{m} 0 \pm 0.1$ (Reddy and Hrivnak 1999). It should be kept in mind, however, that the OI triplet strength also depends on the star effective temperature (Kovtyukh et al. 2012). For 

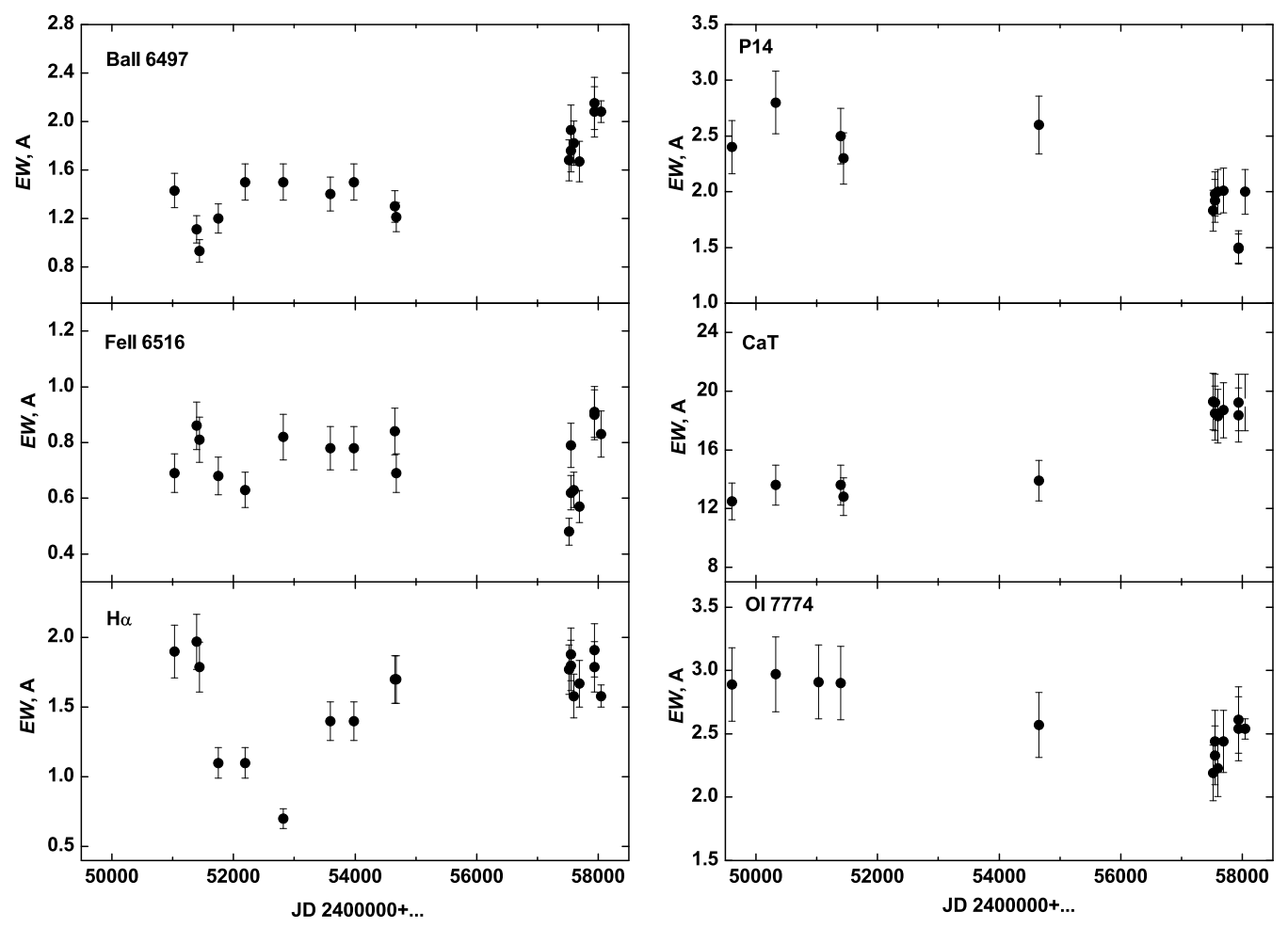

Figure 10: Plot showing evolution of the absorption line equivalent widths for V1427 Aql. 
example, Pereira and Miranda (2007) demonstrate the $E W(\mathrm{OI})$-spectral class and $E W(\mathrm{OI})$-luminosity class relations showing that $E W(\mathrm{OI})$ takes its maximum value for $\mathrm{F} 0$ supergiants and monotonically decreases for later spectral classes. Thus we may assume that the OI triplet weakening detected in the V1427 Aql spectrum implies the decreasing of stellar temperature since 2008 till 2016.

As could be seen in Table 4, the equivalent widths of Paschen lines in the V1427 Aql spectrum slightly decreased by 2016-2017, while the CaII triplet absorptions turned stronger. The total equivalent width of three lines constituting the CaII triplet was $E W(\mathrm{CaT})=12.9 \pm 0.9 \AA$ in the 1994-2008 interval whereas it grew to $E W(\mathrm{CaT})=18.8 \pm 0.4 \AA$ by $2016-2017$.

\section{SPECTRAL CLASS AND EVOLUTION OF STELLAR PARAME- TERS}

The spectral class for V1427 Aql was determined more than once. The HD catalog gives the G5 class. The star was classified as GIa (Bidelman 1981), G5Ia (Buscombe 1984), G40-Ia (Keenan and McNeil 1989), G5Ia (Hrivnak et al. 1989), F8I (Volk and Kwok 1989), F8/G0Ia (Houk and Swift 1999) in the eighties-nineties of the last century. The spectroscopic atlas of post-AGB stars and related objects (Suárez et al. 2006) classifies V1427 Aql as an F7I star basing on the spectra obtained in June 1995. So, there is a discrepancy in the star spectral classification in literature. It is not clear whether it is due to the real change of spectral class or resulted from the application of different classification criteria to a peculiar star spectrum.

There were several studies that attempted to evaluate the atmospheric parameters for V1427 Aql applying the high-resolution spectra analysis. Zacs et al. (1996) got $T_{\text {eff }}=6800 \mathrm{~K}$, Reddy and Hrivnak (1999) found the $T_{\text {eff }}$ value equal to $6750 \mathrm{~K}$, Thévenin et al. (2000) derived $T_{\text {eff }}=5660 \mathrm{~K}$. Kipper et al. (2008) estimated the effective temperature to be $6750 \pm 200 \mathrm{~K}$. Şahin et al. (2016) basing on their observational data obtained in the 2008-2013 interval derived some of the stellar parameters including those determined earlier by other researchers. In Şahin et al. (2016) the effective temperature was found higher than previously, $T_{\text {eff }}=7350 \pm 200 \mathrm{~K}$, and no systematic change of temperature was detected from 1992 till 2008. Such a high $T_{\text {eff }}$ value leads to about F0 spectral class in the calibration of Straižys (1977).

Reddy and Hrivnak (1999) pointed out the difference for V1427 Aql between the spectral class derived from low-resolution spectra and the effective temperature yielded by the model atmospheres method operating on high-resolution spectra. New $T_{\text {eff }}$ estimate presented by Şahin et al. (2016) exacerbates this discrepancy.

We estimated the effective temperature and spectral class for V1427 Aql using our low-resolution spectra. Table 4 lists the $T_{\text {eff }}$ values derived from the empirical relations between the ratio of CaII IRtriplet lines equivalent widths to those of hydrogen Paschen lines (P12, P14, and P17) and the effective temperature (equation (1) from Mantegazza (1991)). Our observational data yield an average $T_{\text {eff }}$ value of $6870 \pm 180 \mathrm{~K}$ for the $1994-2008$ interval. The temperature decreased to $T_{\text {eff }}=5990 \pm 100 \mathrm{~K}$ by 2016-2017. According to the Straižys (1977) calibration for supergiants, these temperatures correspond to F2-F5 spectral classes in 1994-2008 and about F8 in 2016-2017.

A grid of stellar parameters as a function of $\mathrm{CaT}^{*}$ and PaT created from the Cenarro et al. (2002) study is adduced in a paper that aims to search for yellow supergiants in the M33 galaxy (Kourniotis et al. 2017). The PaT index is equal to the sum of P12, P14, and P17 equivalent widths, whereas

the $\mathrm{CaT}^{*}$ index (Cenarro et al. 2001) measures the equivalent width of the integrated CaII triplet corrected for the contamination from the adjacent Paschen lines: $\mathrm{CaT}^{*}=\mathrm{CaT}-0.93 \times \mathrm{PaT}$. If to plot our measurements for V1427 Aql in the PaT-CaT* plane, two states can be distinguished for the star: that with larger values of $T_{\text {eff }}$ and $\log g$ observed in 1994-2008 and the other with smaller ones taking 


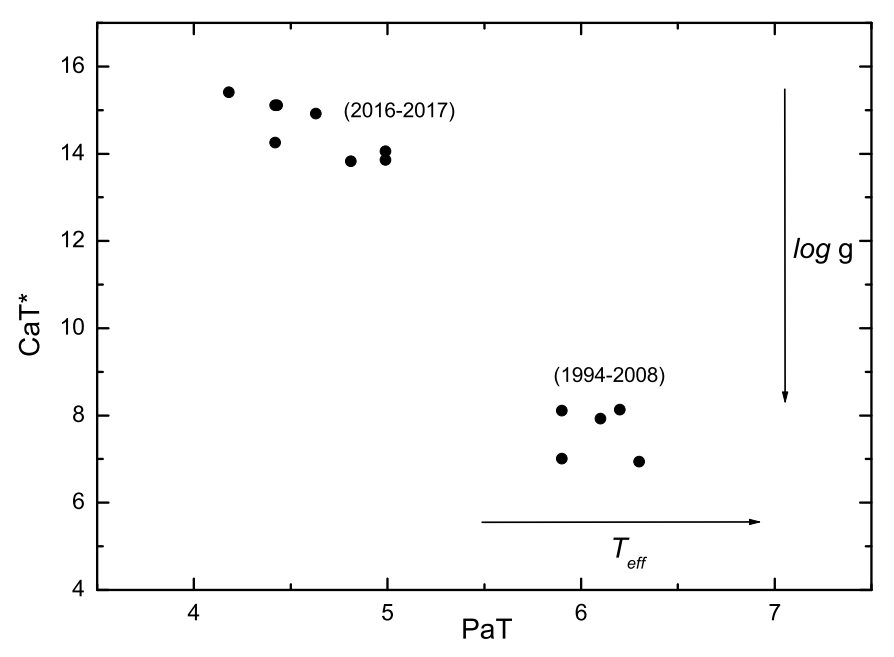

Figure 11: PaT-CaT* diagnostic plot for V1427 Aql involving observations of the 1994-2008 and 20162017 intervals. The PaT and $\mathrm{CaT}^{*}$ indices characterize the strength of Paschen lines P12, P14, P17 and of the CaII IR-triplet absorptions, respectively. Arrows indicate the direction of stellar parameters, $\log g$ and $T_{\text {eff }}$, growing.

place in 2016-2017 (Fig. 11).

We also estimated $T_{\text {eff }}$ from our photometric data. For this purpose the annual average $B-V$ color values corrected for interstellar reddening with $E(B-V)=0 \cdot^{m} 7$ were converted to $T_{\text {eff }}$ according to the $(B-V)-T_{\text {eff }}$ relation for normal supergiants from Flower (1996). Besides we calculated $T_{\text {eff }}$ using the $(V-K)-T_{\text {eff }}$ relation from Bessel et al. (1998). Fig. 12 shows the temporary temperature change. $T_{\text {eff }}$ displays a clear tendency to increase since the first observations in $1990(\sim 5500 \mathrm{~K})$ reaching the maximum value $(\sim 6000 \mathrm{~K})$ in the $2001-2003$ interval and to decrease later on to $\sim 5300$ $\mathrm{K}$. What provokes concern is that the estimates derived from optical observations start to diverge from those yielded by near-IR data after the year 2014 .

The effective temperature estimated from the optical and near-IR photometry is slightly lower than that derived from spectral observations. This difference may be due to the fact that the "photometric" temperature should reflect the state of the so-called "pseudo-photosphere", i.e., of the continuum forming region in the stellar wind, whereas the "spectral" temperature refers to the absorption forming zone, as well as to the possible underestimate of interstellar reddening. If $E(B-V)=1 .{ }^{m} 0$ is adopted for the object, the "photometric" and "spectral" temperatures will converge. We should say that interstellar extinction for HD 179821 has been determined more than once and the $A_{V}$ value given by different authors varies from $1^{m}$ to $4^{m}$ (Pottasch and Parthasarathy 1988; Reddy and Hrivnak 1999; Arkhipova et al. 2001; Arkhipova et al. 2009).

We have also measured the annual average bolometric brightness for V1427 Aql $m_{b o l}=V-A(V)+$ $B C(V)$ and $m_{b o l}=K-A(K)+B C(K)$ using the $B C(V)$ and $B C(K)$ bolometric corrections from Bessel et al. (1998) and adopting $A(V)=3.1 \times E(B-V)$ and $A(K)=0.28 \times E(B-V)$ according to Koornneef (1983). The upper panel of Fig. 12 displays the $m_{b o l}$ change with time. The bolometric magnitude varies over a range of $0 .{ }^{m} 3$ that corresponds to a $\sim 30 \%$ change in luminosity.

Photometric data evidence that the photosphere temperature decreased by $\sim 400 \mathrm{~K}$ between 2008 and 2016, the luminosity grew by $\sim 19 \%$, and the radius increased by $\sim 24 \%$ according to the Stefan-Boltzmann law. 


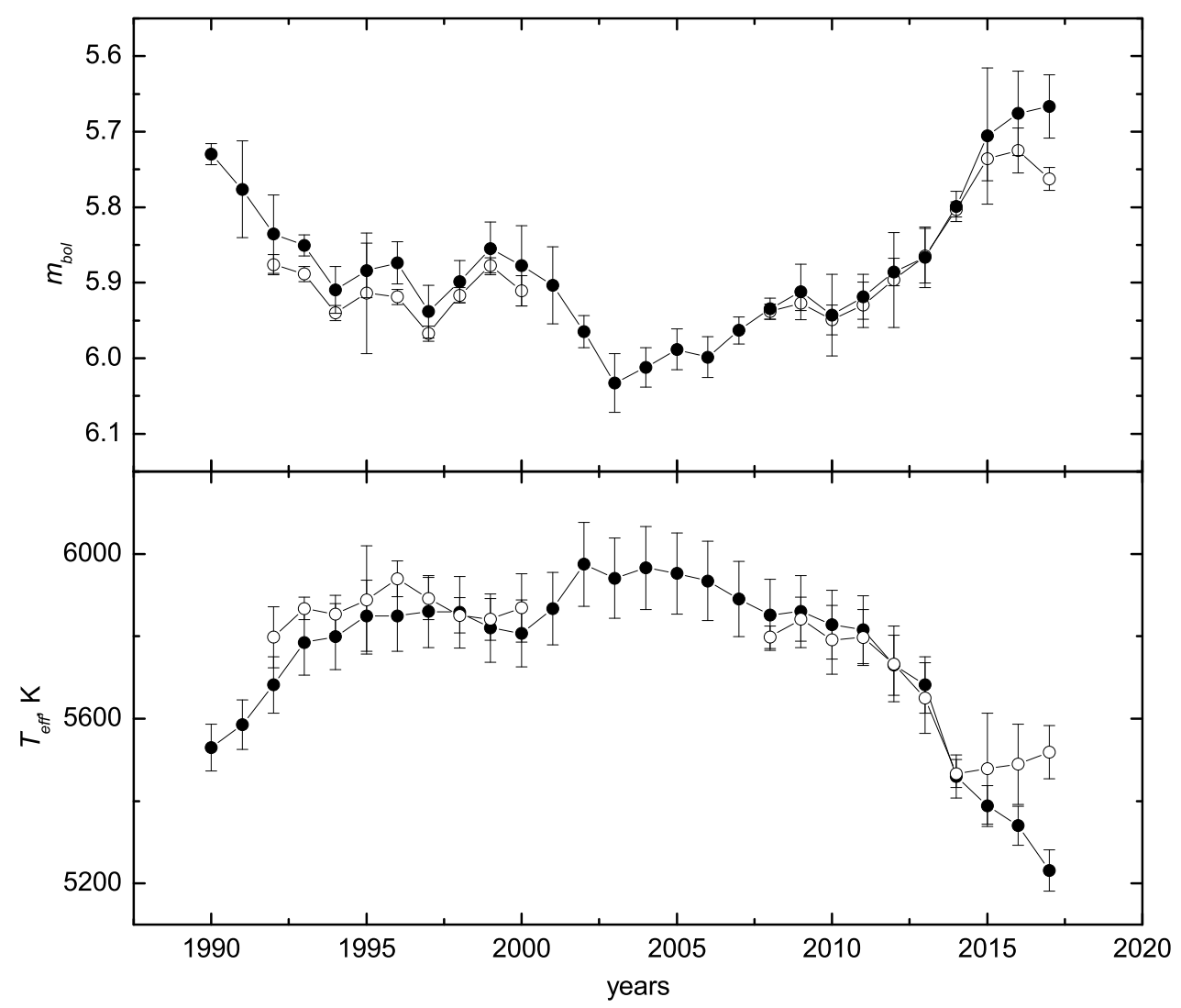

Figure 12: Change of effective temperature and bolometric brightness with time for V1427 Aql. Upper panel: bolometric magnitudes derived from $m_{b o l}=V-A(V)+B C(V)$ are plotted with points, open circles correspond to $m_{b o l}=K-A(K)+B C(K)$. Lower panel: points connected by a line represent the $T_{\text {eff }}$ estimates derived from the $(B-V)-T_{\text {eff }}$ for normal supergiants given in Flower (1996). Open circles correspond to effective temperatures inferred by the $(V-K)-T_{\text {eff }}$ relation of Bessel et al. (1998). 


\section{DISCUSSION}

Owing to the multi-year photometric monitoring of V1427 Aql, we can conclude that the star displays a semi-periodic variation and a long-term trend in brightness and color as well. Spectral and photometric data make it evident that the stellar parameters have changed in a short time.

Let us try to explain these changes with some assumptions made about the object's nature.

The star has already passed through a considerable part of its evolution that is implied by the presence of an expanded nearly spherical gas-dust circumstellar envelope. V1427 Aql has a dual-peak energy distribution in the $\lambda \lambda 0.4-100 \mu \mathrm{m}$ wavelength range that is characteristic of post-AGB objects (Pottasch and Parthasarathy 1988; Hrivnak et al. 1989). The structures that provide the primary input into the energy emitted by the system are the stellar photosphere and the cold dust envelope with a temperature of $T_{d}=128 \mathrm{~K}$ (Odenwald 1986).

According to theoretical simulations, the stars with low and intermediate masses having left the Asymptotic Giant Branch (AGB) continue to evolve to higher temperatures with the luminosity staying on a constant level. New photometric and spectral data for V1427 Aql demonstrate that in recent years a rapid cooling of photosphere has occurred, the star luminosity has grown, and the timescale of semi-periodic variation has increased, and all this does not agree with a model track for a post-AGB object.

A compelling argument for the post-AGB nature of V1427 Aql consists in a specific abundance pattern derived in Zacs et al. (1996), Reddy and Hrivnak (1999), Thévenin et al. (2000), Kipper (2008). The authors found overabundances of carbon, oxygen, nitrogen and s-process elements as compared to solar element distribution, - a pattern that is typical of post-AGB objects. But the recent study of Şahin et al. (2016) states that the stellar atmosphere is not enhanced in s-process elements and that the luminosity, effective temperature, and surface gravity of V1427 Aql are those of a massive star evolving to become a red supergiant and to explode, in time, as a Supernova II.

Earlier investigations have provided some other marks that V1427 Aql is a massive object with increased luminosity. For example, the CO envelope expands with a velocity of $V_{\text {exp }}=33.9 \mathrm{~km} / \mathrm{s}$ (Zuckerman and Dyck) that is noticeably larger than $10-15 \mathrm{~km} / \mathrm{s}$ that is typical of post-AGB stars. The OI triplet at $\lambda 7774 \AA$ in the V1427 Aql spectrum is reported to have an equivalent width in the range of 2.3-3.0 $\AA$ according to different estimates, and this value corresponds to the absolute magnitude of $M_{b o l}<-8 \cdot^{m} 0$ and the luminosity of $L>10^{5} L \odot$. Such a luminosity and the derived temperature place the star into the region on the Hertzsprung-Russell (HR) diagram occupied by yellow hypergiants (YHGs). The YHG region is bounded on the side of higher temperatures by a so-called Yellow Evolutionary Void firstly proposed and described in Nieuwenhuijzen and de Jager (1995). Episodes of large-scale mass loss occurring in this phase of evolution make the stars move from red to blue and vice versa in the HR diagram more than once keeping the bolometric luminosity nearly constant. After the star evolves off the RSG and reaches a temperature of about $7000 \mathrm{~K}$, an extensive mass loss ensues, the star is surrounded by a cool photosphere and starts to move to the RSG region. The envelope turning optically thin, the star goes to the blue in the HR diagram (Oudmaijer et al. 2009). The photometric behavior is seen to be in line with this scenario.

We would like to compare V1427 Aql with other well-studied hypergiants: $\rho$ Cas, V509 Cas, V1302 Aql. All these stars display a significant variation in the spectrum and stellar parameters.

The most famous object, IRC+10 420 (V1302 Aql), which is a strong IR-source, was found to have increased effective temperature by $\sim 1000 \mathrm{~K}$ in 20 years since the seventies of the last century (Oudmaijer et al. 1996) and to have changed its spectral class from F (Humphreys et al. 1973) to A5 (Oudmaijer et al. 1996; Klochkova et al. 1997; Klochkova et al. 2002). The stellar temperature was reported to increase at a rate of $120 \mathrm{~K}$ per year which caused the star to approach the Wolf-Rayet phase (Klochkova et al. 2002; Humphreys et al. 2002). Continuing to monitor V1302 Aql in 2001-2014 
Klochkova et al. (2016) conclude that the hypergiant has entered the phase, during which the effective temperature growth slows down (or ceases), and it is located close to the high-temperature boundary of the Yellow Void in the HR diagram.

$\rho$ Cas, a prototype of YHGs, displayed a significant variation in brightness and spectrum but of another kind than V1302 Aql (Halbedel 1991). The star is known to have experienced three episodes of extensive mass loss during the last 100 years: in 1945-1947, in 1985-1986, and in 2000-2001, the latter was described in detail in Lobel et al. (2003). From the June to September of 2000 the star brightness decreased by nearly $1^{m}$, and the star got out of minimum by the April of 2001. The TiO bands appeared in the spectrum in the summer of 2000; the spectral modeling revealed that the temperature had decreased by at least $3000 \mathrm{~K}$, and the mass loss rate during that episode was estimated to be $\dot{M} \simeq 5.4 \times 10^{-2} M_{\odot}$. The star is considered to lose mass extremely rapidly in the course of the so-called "shell episodes" and to appear wrapped for several hundreds of days in a pseudo-photosphere formed by ejected cool matter. Klochkova et al. (2014) have studied in detail the optical spectrum and have picked out kinematic properties of the expanded stellar atmosphere.

The photometric history was monitored for V509 Cas for a hundred and fifty years (Zsoldos 1986a; Percy and Zsoldos 1992; Halbedel 1993; Nieuwenhuijzen et al. 2012). V509 Cas was observed to change brightness and color monotonically (Percy and Zsoldos 1992; Nieuwenhuijzen et al. 2012). Nieuwenhuijzen et al. (2012) showed that the effective temperature, $\log g$, radius, and luminosity of the star had changed a lot with time. The $\log g$ and turbulent velocity $\xi_{t}$ variation argues that the star has undergone a series of subsequent gas outflows. During every outflow, the pseudo-photosphere happens to have smaller $\log g$ and larger $\xi_{t}$. After the expelled envelope is dispersed into space and the stellar atmosphere is adjusted, a more compact and hot atmosphere is seen.

Let us consider the pulsation activity of the stars above-mentioned.

There is no data evidencing the periodic light variation for V1302 Aql; the star shows only monotonic long-term trends in brightness.

V509 Cas displays semi-periodic variation with a period of $\sim 400^{d}$ and an amplitude up to $0 .^{m} 2$ (Zsoldos 1986b). Percy and Zsoldos (1992) found three peaks in the frequency spectrum at $P=203 .{ }^{d} 3$, 299. ${ }^{d} 2$, and $385 .{ }^{d} 4$ and a tendency for the fundamental mode amplitude to decrease along with the $B-V$ color getting bluer.

The variable star $\rho$ Cas, apart from dimming by more than $1^{m}$ as described above, shows semiperiodic variation with smaller amplitude. Arellano Ferro (1985), based on 1964-1981 observations, found a period of $483 .^{d} 5$.

The object under study, V1427 Aql, displayed a semi-periodic brightness variation with periods in the range $170-200^{d}$ and with the timescale and the amplitude of the oscillation increasing in recent years. The star undergoes temperature variation as well as brightness change with no apparent change of color.

Fadeev (2011) in his paper devoted to the simulations of yellow hypergiants pulsational instability has referred to V1427 Aql as to an object that can not be a hypergiant because of its too long variation period that is larger than the uppermost value predicted by the radial pulsation theory for a yellow hypergiant, on the one hand, and because of its average effective temperature $T_{\text {eff }}=6750 \mathrm{~K}$ (Kipper 2008) corresponding to the region of pulsational stability, on the other. On this basis, the author suggests that V1427 Aql is a post-AGB object.

However, it should be noted that oscillation periods for known post-AGB stars in the range of spectral classes from F0 to G5 are not larger than $150^{d}$ (Arkhipova et al. 2003; Arkhipova et al. 2010; Hrivnak et al. 2010; Hrivnak et al. 2015). Besides, the true hypergiants $\rho$ Cas and V509 Cas vary with periods $>200^{d}$ having temperatures $T_{\text {eff }}$ for which radial pulsations are forbidden by theory. Arellano Ferro (1985) suggested that $\rho$ Cas and V509 Cas are non-radial pulsators. The same mechanism may appear responsible for the semi-periodic variation of V1427 Aql. 
So, analyzing the photometric and spectral variability of V1427 Aql showed that the star's behavior is similar to that of yellow hypergiants located near the very unstable Yellow Void and differs significantly from ordinary post-AGB objects.

\section{CONCLUSION}

New photometry and spectra obtained for the yellow hypergiant with a gas-dust envelope, V1427 Aql, and their comparison with archival data lead us to the following results.

1) Variation periods $P=170^{d}$ and $P=141^{d}$ (or $P=217^{d}$ ) were found on the basis of $U B V$ observations in 2009-2011. It is shown that oscillations became less regular after 2011, the characteristic time of brightness variation increased to $260^{d}$ in $2012-2014$, and to $370^{d}$ in the 2015-2017 interval.

2) The annual average brightness was found to gradually increase in 2009-2015 in the $V, J, K$ filters, and to decrease in $U$ and $B$. The annual average $U-B, B-V, J-K$ colors were growing (the star was getting redder). The near-IR brightness stopped increasing in 2016-2017, and V1427 Aql showed a tendency to get bluer, whereas it kept on getting redder in the optical.

3) The long-term trend in colors was proved to reflect the change of photosphere temperature, increasing since 1990 till 2002 and then systematically decreasing. Long-term variation of the bolometric magnitude does not agree with the assumption of quick evolution at constant luminosity but seems to be related to mass loss episodes that result in the expanded stellar envelope getting optically thicker and the continuum spectrum originating in more outer and cool layers.

4) The comparison of the 2016-2017 spectral data for V1427 Aql with those obtained in 1994-2008 revealed the strengthening of $\mathrm{BaII}$ and CaII IR-triplet absorptions and the weakening of OI triplet blend. These changes are supposed to be due to the decreasing of temperature in the region where the absorptions are formed.

It is very important to continue photometric and spectral observations of the object that changes so quickly. It is highly recommended to carry out both photometric and spectral monitoring of the star with no durable gaps.

\section{ACKNOWLEDGMENT}

This study has made use of the SIMBAD and VIZIER databases and the NASA ADS.

\section{REFERENCES}

1. A. Arellano Ferro, MNRAS 216, 571 (1985).

2. V.P. Arkhipova, N.P. Ikonnikova, R.I. Noskova, Astron Lett. 19, 169 (1993).

3. V.P. Arkhipova, N.P. Ikonnikova, R.I. Noskova, G.V. Sokol, S.Yu. Shugarov, Astron. Lett. 27, 156 (2001).

4. V.P. Arkhipova, R.I. Noskova, N.P. Ikonnikova, G.V. Komissarova, Astron. Lett. 29, 480 (2003).

5. V.P. Arkhipova, V.P.Esipov, N.P. Ikonnikova, G.V. Komissarova, A.M. Tatarnikov, B.F. Yudin, Astron. Lett. 35, 764 (2009).

6. V.P. Arkhipova, N.P. Ikonnikova, G.V. Komissarova, Astron. Lett. 36, 269 (2010).

7. M.S. Bessell, F. Castelli, P. Planesas, Astron. Astrophys. 333, 231 (1998).

8. W.P.Bidelman, Astron. J. 86, 553 (1981). 
9. W. Buscombe, MK Spectral Classification, Sixth General Catalog (Evanston: Northwestern University, 1984).

10. A.J. Cenarro, P.N. Cardiel, J.Gorgas, R.F. Peletier, A.Vazdekis, and F. Prada, MNRAS 326, 959 (2001).

11. A. J. Cenarro, J. Gorgas, N. Cardiel, A. Vazdekis, and R.F. Peletier, MNRAS 329, 863 (2002).

12. H. Coroller, A. Lébre, D. Gillet, and E. Chapellier, Astron. Astrophys. 400, 613 (2003).

13. T. J. Deeming, Astrophys. and Space Sci. 36, 137 (1975).

14. Yu.A. Fadeev, Astron. Lett. 37, 403 (2011).

15. B.A. Ferguson and T. Ueta, Astrophys. J. 711, 613 (2010).

16. P.J. Flower, Astrophys. J. 469, 355 (1996).

17. I.N. Glushneva, V.T. Doroshenko, T.S. Fetisova, T.S. Khruzina, E.A. Kolotilov, L.V. Mossakovskaya, S.L. Ovchinnikov, and I.B.Voloshina, VizieR Online Data Catalog III/208 (1998).

18. E.M. Halbedel, IBVS No. 3616, 1 (1991).

19. E.M. Halbedel, IBVS No. 3849, 1 (1993).

20. N. Houk and C. Swift, Michigan catalogue of two-dimensional spectral types for the HD Stars vol. 5. By Nancy Houk and Carrie Swift (Ann Arbor, Michigan: Department of Astronomy, University of Michigan, 1999).

21. B.J. Hrivnak, S. Kwok, and K.M. Volk, Astrophys. J. 346, 265, (1989).

22. B.J. Hrivnak, in Post-AGB Objects as a Phase of Stellar Evolution, Proceedings of the Torun Workshop held July5-7, 2000. Ed. R. Szczerba and S.K. Górny, Astroph. Space Sci. Library 265 (Kluwer Academic Pub, 2001), p. 101

23. B.J. Hrivnak, W. Lu, R.E. Maupin, and B.D. Spitzbart, Astrophys. J. 709, 1042 (2010).

24. B.J. Hrivnak, W. Lu, and K. A. Nault, Astron. J. 149, 184 (2015).

25. R.M. Humphreys, D.W. Strecker, T.L. Urdock, and F.J. Low, Astrophys. J. 179, L49-L52 (1973).

26. R.M. Humphreys, K. Davidson, and N. Smith, Astron. J. 1724, 1026 (2002).

27. H.L. Johnson, R.I. Mitchel, B. Iriarte, and W.Z. Wisniewski, Comm. Lunar and Planet. Lab. 4, 99 (1966).

28. E. Josselin and A. Lebre, Astron. Astrophys. 367, 826 (2001).

29. M. Jura, T. Velusamy, and M.W. Werner, Astrophys. J. 556, 408 (2001).

30. J.H. Kastner and D.A. Weintraub, Astrophys. J. 452, 833 (1995).

31. P.C. Keenan and R.C. McNeil, Astrophys. J. Suppl. Ser. 71, 245 (1989).

32. T. Kipper, Baltic Astronomy 17, 87 (2008). 
33. V. Klochkova, E.L. Chentsov, and V. Panchuk, MNRAS 292, 19 (1997).

34. V.G. Klochlova, M.V. Yushkin, E.L. Chentsov, and V.E. Panchuk, Astron. Rep. 46, 139 (2002).

35. V.G. Klochkova, Astrophys. Bull. 69, 279 (2014).

36. V.G. Klochlova, V.E. Panchuk, N.S. Tavolzhanskaya, and I.A. Usenko, Astron. Rep. 58, 101 (2014).

37. V.G. Klochkova, E.L. Chentsov, A.S. Miroshnichenko, V.E. Panchuk, and M.V. Yushkin, MNRAS 459, 4183 (2016).

38. J. Koornneef, Astron. Asrophys. 128, 84 (1983).

39. M. Kourniotis, A. Z. Bonanos, W. Yuan, L. M. Macri, D. Garcia-Alvarez, and C.-H. Lee, Astron. Astrophys. 601, id.A76, 21 (2017).

40. V. V. Kovtyukh, N. I. Gorlova, and S. I. Belik, MNRAS 423, 3268 (2012).

41. P. Lenz and M. Breger, Communications in Asteroseismology, 146, 53 (2005).

42. A. Lobel, A. K. Dupree, R. P. Stefanik, G. Torres, G. Israelian, N. Morrison, C. de Jager, H. Nieuwenhuijzen, I. Ilyin, and F. Musaev, Astrophys. J. 583, 923 (2003).

43. V. M. Lyutyi, Soobshch. GAISh 172, 30 (1971).

44. L. Mantegazza, Astron. Astrophys. Suppl. 88, 255 (1991).

45. H. Nieuwenhuijzen and C. de Jager, Astron. Astrophys. 302, 811 (1995).

46. H. Nieuwenhuijzen, C. De Jager, I. Kolka, G. Israelian, A. Lobel, E. Zsoldos, A. Maeder, and G. Meynet, Astron. Astrophys. 546, A105 (2012).

47. S.F. Odenwald, Astrophys. J. 307, 714 (1986).

48. R.D. Oudmaijer, M.A.T. Groenewegen, H.E. Matthews, J.A.D.L. Blommaert, and K.C. Sahu, MNRAS 280, 1062 (1996).

49. R.D. Oudmaijer, B.Davies, W.-J. de Wit, and M. Patel, in The Biggest, Baddest, Coolest Stars, ASP Conference Series, Vol. 412, Proceedings of the workshop held 16-18 July 2007, at the Millennium Centre, Johnson City, Tennessee, USA. Ed. by D.G. Luttermoser, B.J. Smith, and R.E. Stencel (San Francisco: Astronomical Society of the Pacific, 2009), p.17.

50. J.R. Percy and E. Zsoldos, Astron. Astrophys., 263, 123 (1992).

51. C.B. Pereira and L.F. Miranda, Astron. Astrophys. 462, 231 (2007).

52. A.J. Pickles, PASP 110, 863 (1998).

53. D. Pooley, Thesis, University of Canterbury (2003).

54. S.R. Pottasch and . Parthasarathy, Astron. Astrophys. 192, 182 (1988).

55. B.E. Reddy and B.J. Hrivnak, Astron. J. 117, 1834 (1999).

56. T. Şahin, D.L. Lambert, V.G. Klochkova, and V.E. Panchuk, MNRAS 461, 4071 (2016). 
57. N.N. Samus, E.V. Kazarovets, O.V. Durlevich, N.N. Kireeva, and E.N. Pastukhova, Astron. Rep. 61, 80 (2017).

58. C. Sánchez Contreras, R. Sahai, A. Gil de Paz, and R. Goodrich, Astrophys. J. Suppl. Ser. 179, 166 (2008).

59. S.G. Sergeev and F. Heisberger, A Users Manual for SPE. Wien (1993).

60. V.I. Shenavrin, O.G. Taranova, and A. E. Nadzhip, Astron, Rep. 55, 31 (2011).

61. V.L. Straižys, Mnogotsvetnaya fotometriya zvezd (Vilnius: Mokslas, 1977).

62. O. Suarez, J.F. Gomez, and O. Morata, Astron. and Astrophys. 458, 173 (2006).

63. S. Tamura, M. Takeuti, IBVS No. 3561 (1991).

64. F. Thévenin, M. Parthasarathy, and G. Jasniewicz, Astron. Astrophys. 359, 138 (2000).

65. W.E.C.J. van der Veen, H.J. Habing and T.R. Geballe, Astron. Astrophys. 226, 108 (1989).

66. F. van Leeuwen, Astron. Astrophys. 474, 653 (2007).

67. K.M. Volk and S. Kwok, Astrophys. J. 342, 345 (1989).

68. L. Zacs, V.G. Klochkova, V.E. Panchuk, and R. Spelmanis, MNRAS 282, 1171 (1996).

69. E. Zsoldos, The Observatory 106, 156 (1986a).

70. E. Zsoldos, in Luminous Stars and Associations in Galaxies, Proceedings of the IAU Symposium No. 116. Ed. by de Loore C.W.H., Willis A.J., Laskarides P. (Reidel Publ. Co., Dordrecht, 1986b), p. 87.

71. B. Zuckerman and H.M. Dyck, Astrophys. J. 311, 345 (1986). 
Table 1: $U B V$-photometry for V1427 Aql in 2009-2017.

\begin{tabular}{cccccc}
\hline JD & $V$ & $B$ & $U$ & $B-V$ & $U-B$ \\
\hline 2454970 & 8.030 & 9.331 & 10.453 & 1.301 & 1.122 \\
2454972 & 8.021 & 9.345 & 10.461 & 1.323 & 1.116 \\
2454978 & 8.022 & 9.331 & 10.435 & 1.309 & 1.105 \\
2454981 & 8.030 & 9.341 & 10.387 & 1.310 & 1.046 \\
2454983 & 8.048 & 9.343 & 10.361 & 1.296 & 1.018 \\
2455002 & 8.064 & 9.383 & 10.532 & 1.319 & 1.149 \\
2455005 & 8.054 & 9.358 & 10.432 & 1.305 & 1.073 \\
2455010 & 8.055 & 9.364 & 10.490 & 1.309 & 1.126 \\
2455031 & 8.093 & 9.467 & 10.557 & 1.374 & 1.089 \\
2455032 & 8.113 & 9.427 & 10.574 & 1.314 & 1.147 \\
2455036 & 8.096 & 9.416 & 10.591 & 1.320 & 1.175 \\
2455041 & 8.097 & 9.416 & 10.556 & 1.319 & 1.140 \\
2455043 & 8.067 & 9.431 & 10.635 & 1.364 & 1.204 \\
2455055 & 8.063 & 9.369 & 10.516 & 1.306 & 1.147 \\
2455061 & 8.058 & 9.369 & 10.486 & 1.310 & 1.118 \\
2455065 & 8.052 & 9.363 & 10.448 & 1.310 & 1.085 \\
2455069 & 8.032 & 9.345 & 10.446 & 1.312 & 1.101 \\
2455071 & 8.032 & 9.335 & 10.445 & 1.303 & 1.110 \\
2455073 & 8.046 & 9.345 & 10.459 & 1.299 & 1.114 \\
2455086 & 8.004 & 9.287 & 10.374 & 1.283 & 1.087 \\
2455090 & 7.996 & 9.267 & 10.331 & 1.271 & 1.065 \\
2455093 & 7.985 & 9.274 & 10.314 & 1.289 & 1.040 \\
2455098 & 7.983 & 9.263 & 10.372 & 1.280 & 1.109 \\
2455103 & 8.003 & 9.281 & 10.399 & 1.278 & 1.118 \\
2455112 & 7.993 & 9.287 & 10.384 & 1.295 & 1.097 \\
2455113 & 7.986 & 9.279 & 10.393 & 1.293 & 1.114 \\
2455118 & 8.007 & 9.268 & 10.381 & 1.261 & 1.113 \\
2455145 & 8.014 & 9.298 & 10.381 & 1.284 & 1.083 \\
2455151 & 8.014 & 9.318 & 10.368 & 1.305 & 1.049 \\
2455159 & 8.068 & 9.355 & 10.390 & 1.287 & 1.034 \\
2455164 & 8.066 & 9.370 & 10.450 & 1.304 & 1.081 \\
2455281 & 8.021 & 9.325 & 10.419 & 1.305 & 1.094 \\
2455291 & 8.037 & 9.337 & 10.458 & 1.300 & 1.121 \\
2455305 & 8.080 & 9.391 & 10.526 & 1.310 & 1.135 \\
2455313 & 8.079 & 9.403 & 10.546 & 1.323 & 1.143 \\
2455315 & 8.092 & 9.422 & 10.619 & 1.330 & 1.198 \\
2455329 & 8.121 & 9.470 & 10.666 & 1.349 & 1.196 \\
2455330 & 8.137 & 9.475 & 10.641 & 1.338 & 1.166 \\
2455341 & 8.121 & 9.468 & 10.642 & 1.347 & 1.174 \\
2455344 & 8.144 & 9.479 & 10.655 & 1.335 & 1.176 \\
2455349 & 8.117 & 9.479 & 10.645 & 1.362 & 1.165 \\
2455357 & 8.109 & 9.447 & 10.553 & 1.338 & 1.106 \\
2455358 & 8.086 & 9.439 & 10.576 & 1.353 & 1.137 \\
2455364 & 8.115 & 9.443 & 10.610 & 1.328 & 1.167 \\
2455369 & 8.092 & 9.411 & 10.573 & 1.319 & 1.162
\end{tabular}


continued Table 1

\begin{tabular}{cccccc}
\hline JD & $V$ & $B$ & $U$ & $B-V$ & $U-B$ \\
\hline 2455380 & 8.075 & 9.399 & 10.499 & 1.323 & 1.100 \\
2455389 & 8.042 & 9.345 & 10.494 & 1.303 & 1.149 \\
2455408 & 7.989 & 9.277 & 10.391 & 1.288 & 1.114 \\
2455418 & 7.987 & 9.259 & 10.358 & 1.272 & 1.099 \\
2455424 & 7.979 & 9.258 & 10.335 & 1.279 & 1.076 \\
2455445 & 8.001 & 9.290 & 10.391 & 1.289 & 1.101 \\
2455448 & 8.009 & 9.288 & 10.423 & 1.279 & 1.135 \\
2455454 & 8.019 & 9.318 & 10.456 & 1.299 & 1.138 \\
2455463 & 8.035 & 9.335 & 10.494 & 1.300 & 1.159 \\
2455468 & 8.054 & 9.357 & 10.488 & 1.303 & 1.132 \\
2455492 & 8.108 & 9.440 & 10.622 & 1.333 & 1.181 \\
2455494 & 8.118 & 9.454 & 10.632 & 1.336 & 1.178 \\
2455501 & 8.131 & 9.491 & 10.662 & 1.359 & 1.172 \\
2455507 & 8.137 & 9.482 & 10.681 & 1.346 & 1.199 \\
2455516 & 8.125 & 9.457 & 10.617 & 1.332 & 1.161 \\
2455678 & 8.072 & 9.403 & 10.540 & 1.331 & 1.137 \\
2455688 & 8.074 & 9.406 & 10.576 & 1.333 & 1.169 \\
2455692 & 8.074 & 9.404 & 10.572 & 1.330 & 1.168 \\
2455697 & 8.074 & 9.404 & 10.562 & 1.330 & 1.159 \\
2455708 & 8.072 & 9.403 & 10.540 & 1.331 & 1.137 \\
2455715 & 8.074 & 9.406 & 10.576 & 1.333 & 1.169 \\
2455720 & 8.074 & 9.404 & 10.572 & 1.330 & 1.168 \\
2455734 & 8.074 & 9.404 & 10.562 & 1.330 & 1.159 \\
2455737 & 8.076 & 9.397 & 10.573 & 1.321 & 1.176 \\
2455746 & 8.083 & 9.407 & 10.568 & 1.323 & 1.162 \\
2455752 & 8.076 & 9.401 & 10.551 & 1.325 & 1.150 \\
2455763 & 8.060 & 9.376 & 10.525 & 1.316 & 1.149 \\
2455765 & 8.072 & 9.392 & 10.560 & 1.320 & 1.168 \\
2455768 & 8.046 & 9.378 & 10.517 & 1.332 & 1.139 \\
2455774 & 8.047 & 9.364 & 10.496 & 1.317 & 1.132 \\
2455780 & 8.033 & 9.348 & 10.518 & 1.315 & 1.169 \\
2455782 & 8.031 & 9.344 & 10.521 & 1.312 & 1.177 \\
2455795 & 8.040 & 9.356 & 10.521 & 1.316 & 1.165 \\
2455810 & 8.009 & 9.332 & 10.480 & 1.322 & 1.149 \\
2455823 & 7.995 & 9.308 & 10.488 & 1.312 & 1.180 \\
2455832 & 7.989 & 9.300 & 10.465 & 1.310 & 1.165 \\
2455841 & 8.011 & 9.327 & 10.513 & 1.316 & 1.186 \\
2455861 & 8.087 & 9.464 & 10.591 & 1.377 & 1.127 \\
2455863 & 8.044 & 9.379 & 10.550 & 1.335 & 1.170 \\
2455866 & 8.061 & 9.396 & 10.557 & 1.335 & 1.161 \\
2455873 & 8.077 & 9.422 & 10.608 & 1.346 & 1.186 \\
2455900 & 8.105 & 9.429 & 10.561 & 1.323 & 1.133 \\
2456040 & 8.038 & 9.369 & 10.529 & 1.331 & 1.161 \\
2456043 & 8.037 & 9.375 & 10.505 & 1.338 & 1.130 \\
2456046 & 8.043 & 9.402 & 10.572 & 1.358 & 1.170 \\
2456051 & 8.048 & 9.405 & 10.592 & 1.357 & 1.187
\end{tabular}


continued Table 1

\begin{tabular}{cccccc}
\hline JD & $V$ & $B$ & $U$ & $B-V$ & $U-B$ \\
\hline 2456059 & 8.031 & 9.398 & 10.602 & 1.367 & 1.204 \\
2456066 & 8.048 & 9.400 & 10.582 & 1.352 & 1.182 \\
2456093 & 8.069 & 9.411 & 10.624 & 1.342 & 1.214 \\
2456098 & 8.062 & 9.416 & 10.629 & 1.354 & 1.213 \\
2456103 & 8.050 & 9.411 & 10.632 & 1.360 & 1.221 \\
2456118 & 8.042 & 9.405 & 10.594 & 1.363 & 1.189 \\
2456123 & 8.041 & 9.415 & 10.620 & 1.374 & 1.205 \\
2456124 & 8.040 & 9.418 & 10.616 & 1.378 & 1.199 \\
2456128 & 8.047 & 9.416 & 10.625 & 1.369 & 1.209 \\
2456131 & 8.042 & 9.426 & 10.623 & 1.384 & 1.196 \\
2456151 & 8.053 & 9.433 & 10.650 & 1.380 & 1.217 \\
2456156 & 8.048 & 9.437 & 10.654 & 1.389 & 1.217 \\
2456162 & 8.041 & 9.431 & 10.659 & 1.391 & 1.228 \\
2456176 & 8.029 & 9.402 & 10.608 & 1.373 & 1.206 \\
2456178 & 8.032 & 9.396 & 10.626 & 1.364 & 1.230 \\
2456202 & 8.022 & 9.399 & 10.633 & 1.377 & 1.234 \\
2456224 & 8.003 & 9.379 & 10.578 & 1.376 & 1.199 \\
2456249 & 7.987 & 9.338 & 10.515 & 1.351 & 1.132 \\
2456423 & 8.089 & 9.514 & 10.849 & 1.425 & 1.335 \\
2456434 & 8.088 & 9.487 & 10.776 & 1.399 & 1.289 \\
2456448 & 8.009 & 9.398 & 10.662 & 1.389 & 1.265 \\
2456464 & 7.992 & 9.380 & 10.601 & 1.388 & 1.221 \\
2456472 & 8.020 & 9.405 & 10.625 & 1.385 & 1.220 \\
2456482 & 8.018 & 9.404 & 10.633 & 1.386 & 1.229 \\
2456487 & 8.004 & 9.391 & 10.629 & 1.387 & 1.239 \\
2456511 & 8.012 & 9.388 & 10.598 & 1.376 & 1.210 \\
2456517 & 7.994 & 9.358 & 10.573 & 1.364 & 1.215 \\
2456519 & 7.985 & 9.365 & 10.562 & 1.380 & 1.198 \\
2456531 & 7.985 & 9.365 & 10.577 & 1.380 & 1.213 \\
2456573 & 8.043 & 9.458 & 10.734 & 1.415 & 1.276 \\
2456575 & 8.043 & 9.455 & 10.762 & 1.412 & 1.307 \\
2456605 & 8.050 & 9.475 & 10.774 & 1.425 & 1.299 \\
2456748 & 7.993 & 9.401 & 10.639 & 1.407 & 1.239 \\
2456780 & 8.022 & 9.447 & 10.772 & 1.425 & 1.325 \\
2456832 & 8.026 & 9.516 & 10.876 & 1.490 & 1.360 \\
2456839 & 8.027 & 9.553 & 10.897 & 1.525 & 1.345 \\
2456869 & 8.023 & 9.518 & 10.896 & 1.495 & 1.378 \\
2456884 & 8.028 & 9.574 & 10.951 & 1.547 & 1.377 \\
2456892 & 8.034 & 9.540 & 10.945 & 1.506 & 1.405 \\
2456902 & 8.040 & 9.538 & 10.929 & 1.499 & 1.391 \\
2456917 & 8.020 & 9.501 & 10.841 & 1.481 & 1.340 \\
2456942 & 7.963 & 9.380 & 10.642 & 1.417 & 1.262 \\
2456960 & 7.940 & 9.339 & 10.583 & 1.399 & 1.244 \\
2457162 & 8.047 & 9.580 & 11.018 & 1.533 & 1.439 \\
2457190 & 8.024 & 9.544 & 11.021 & 1.520 & 1.477 \\
2457213 & 8.004 & 9.531 & 10.982 & 1.527 & 1.450
\end{tabular}


continued Table 1

\begin{tabular}{cccccc}
\hline JD & $V$ & $B$ & $U$ & $B-V$ & $U-B$ \\
\hline 2457219 & 7.998 & 9.532 & 10.983 & 1.534 & 1.452 \\
2457225 & 7.978 & 9.507 & 10.948 & 1.529 & 1.441 \\
2457232 & 7.974 & 9.497 & 10.956 & 1.523 & 1.459 \\
2457249 & 7.913 & 9.436 & 10.869 & 1.523 & 1.433 \\
2457269 & 7.844 & 9.324 & 10.781 & 1.480 & 1.457 \\
2457299 & 7.797 & 9.255 & 10.612 & 1.457 & 1.357 \\
2457486 & 8.012 & 9.602 & 11.163 & 1.590 & 1.573 \\
2457495 & 8.012 & 9.588 & 11.163 & 1.576 & 1.575 \\
2457516 & 8.022 & 9.614 & 11.204 & 1.593 & 1.590 \\
2457517 & 8.038 & 9.616 & 11.186 & 1.578 & 1.570 \\
2457525 & 7.984 & 9.575 & 11.152 & 1.591 & 1.577 \\
2457544 & 7.949 & 9.501 & 11.016 & 1.551 & 1.515 \\
2457545 & 7.950 & 9.524 & 11.047 & 1.574 & 1.523 \\
2457549 & 7.955 & 9.499 & 11.015 & 1.545 & 1.515 \\
2457556 & 7.928 & 9.456 & 10.979 & 1.528 & 1.523 \\
2457565 & 7.909 & 9.457 & 10.973 & 1.549 & 1.516 \\
2457573 & 7.909 & 9.451 & 10.943 & 1.527 & 1.469 \\
2457575 & 7.924 & 9.454 & 10.965 & 1.519 & 1.488 \\
2457578 & 7.926 & 9.453 & 10.950 & 1.509 & 1.483 \\
2457581 & 7.926 & 9.452 & 10.929 & 1.549 & 1.413 \\
2457597 & 7.914 & 9.441 & 10.910 & 1.486 & 1.456 \\
2457598 & 7.919 & 9.438 & 10.926 & 1.471 & 1.448 \\
2457602 & 7.896 & 9.405 & 10.888 & 1.481 & 1.443 \\
2457609 & 7.873 & 9.421 & 10.834 & 1.485 & 1.465 \\
2457613 & 7.872 & 9.358 & 10.813 & 1.499 & 1.462 \\
2457625 & 7.886 & 9.357 & 10.805 & 1.471 & 1.448 \\
2457634 & 7.869 & 9.350 & 10.793 & 1.481 & 1.443 \\
2457662 & 7.897 & 9.382 & 10.846 & 1.485 & 1.464 \\
2457665 & 7.897 & 9.395 & 10.858 & 1.498 & 1.462 \\
2457870 & 8.036 & 9.605 & 11.143 & 1.569 & 1.538 \\
2457871 & 8.028 & 9.612 & 11.170 & 1.584 & 1.557 \\
2457905 & 8.016 & 9.603 & 11.152 & 1.587 & 1.549 \\
2457931 & 8.009 & 9.583 & 11.120 & 1.574 & 1.537 \\
2457935 & 8.001 & 9.577 & 11.139 & 1.576 & 1.562 \\
2457950 & 7.993 & 9.576 & 11.135 & 1.584 & 1.559 \\
2457955 & 7.996 & 9.584 & 11.157 & 1.588 & 1.573 \\
2457958 & 7.983 & 9.529 & 11.149 & 1.547 & 1.620 \\
2457961 & 7.998 & 9.591 & 11.180 & 1.594 & 1.589 \\
2457966 & 7.984 & 9.560 & 11.136 & 1.576 & 1.576 \\
2457968 & 7.977 & 9.577 & 11.157 & 1.599 & 1.580 \\
2457977 & 7.971 & 9.529 & 11.132 & 1.558 & 1.603 \\
2457979 & 7.964 & 9.538 & 11.111 & 1.574 & 1.573 \\
2457985 & 7.968 & 9.524 & 11.099 & 1.556 & 1.575 \\
2457990 & 7.941 & 9.506 & 11.069 & 1.565 & 1.563 \\
2458006 & 7.923 & 9.464 & 10.994 & 1.541 & 1.530 \\
2458009 & 7.917 & 9.481 & 11.051 & 1.564 & 1.570
\end{tabular}


continued Table 1

\begin{tabular}{cccccc}
\hline JD & $V$ & $B$ & $U$ & $B-V$ & $U-B$ \\
\hline 2458010 & 7.915 & 9.480 & 11.030 & 1.565 & 1.550 \\
2458015 & 7.907 & 9.461 & 11.015 & 1.554 & 1.554 \\
2458026 & 7.914 & 9.465 & 10.995 & 1.551 & 1.530 \\
2458044 & 7.941 & 9.502 & 11.030 & 1.561 & 1.528 \\
2458075 & 7.981 & 9.566 & 11.175 & 1.585 & 1.608 \\
\hline
\end{tabular}


Table 2: JHKLM-photometry for V1427 Aql in 2009-2017.

\begin{tabular}{cccccc}
\hline JD & $J$ & $H$ & $K$ & $L$ & $M$ \\
\hline 2455048.35 & 5.43 & 5.00 & 4.75 & 4.48 & - \\
2455319.58 & 5.43 & 5.02 & 4.76 & 4.54 & - \\
2455410.34 & 5.36 & - & 4.72 & - & - \\
2455433.31 & 5.39 & - & 4.73 & - & - \\
2455501.15 & 5.45 & - & 4.76 & - & - \\
2455700.53 & 5.40 & - & 4.72 & - & - \\
2455734.45 & 5.44 & - & 4.74 & - & - \\
2455753.40 & 5.44 & - & 4.76 & - & - \\
2455782.33 & 5.40 & 5.00 & 4.73 & 4.50 & - \\
2455818.28 & 5.36 & - & 4.68 & - & - \\
2456084.53 & 5.37 & - & 4.70 & - & - \\
2456147.35 & 5.31 & - & 4.61 & - & - \\
2456409.58 & 5.33 & - & 4.63 & - & - \\
2456470.44 & 5.28 & - & 4.57 & - & - \\
2456489.38 & 5.28 & - & 4.57 & - & - \\
2456515.39 & 5.25 & - & 4.57 & - & - \\
2456591.16 & 5.22 & - & 4.53 & - & - \\
2456876.31 & 5.13 & - & 4.41 & - & - \\
2456968.19 & 5.13 & - & 4.40 & - & - \\
2457233.40 & 5.09 & - & 4.38 & - & - \\
2457261.32 & 5.08 & - & 4.35 & - & - \\
2457266.30 & 5.06 & 4.63 & 4.34 & 4.10 & 3.83 \\
2457292.21 & 5.03 & 4.60 & 4.31 & 4.09 & 3.70 \\
2457525.50 & 5.10 & 4.64 & 4.37 & 4.11 & 4.10 \\
2457529.54 & 5.11 & 4.66 & 4.39 & 4.14 & 4.09 \\
2457556.48 & 5.09 & 4.62 & 4.35 & 4.12 & 4.11 \\
2457566.44 & 5.08 & 4.62 & 4.32 & 4.07 & 3.99 \\
2457583.38 & 5.08 & 4.64 & 4.35 & 4.09 & 4.02 \\
2457594.36 & 5.10 & 4.66 & 4.36 & 4.11 & 4.02 \\
2457616.32 & 5.04 & 4.61 & 4.31 & 4.10 & 3.93 \\
2457640.32 & 5.04 & 4.60 & 4.30 & 4.11 & 3.94 \\
2457855.60 & 5.11 & 4.64 & 4.38 & 4.14 & 4.14 \\
2457892.50 & 5.10 & 4.64 & 4.41 & 4.16 & 4.22 \\
2457909.50 & 5.07 & 4.57 & 4.30 & 4.06 & 3.94 \\
2457912.50 & 5.13 & 4.63 & 4.36 & 4.09 & 3.95 \\
2457943.40 & 5.08 & 4.61 & 4.37 & 4.06 & 4.01 \\
2457948.40 & 5.07 & 4.63 & 4.35 & 4.07 & 4.03 \\
2457950.40 & 5.09 & 4.61 & 4.35 & 4.06 & 3.97 \\
2457971.40 & 5.07 & 4.61 & 4.33 & 4.08 & 4.09 \\
2457975.30 & 5.06 & 4.59 & 4.33 & 4.07 & 4.02 \\
2457978.30 & 5.06 & 4.61 & 4.33 & 4.07 & 4.13 \\
2457999.30 & 5.05 & 4.60 & 4.34 & 4.10 & 3.99 \\
2458008.30 & 5.06 & 4.59 & 4.30 & 4.04 & 4.05 \\
2458063.20 & 5.12 & 4.63 & 4.30 & 4.06 & 4.00 \\
\hline & & & & &
\end{tabular}


Table 3: Log of spectral observations for V1427 Aql in 2016-2017.

\begin{tabular}{ccc}
\hline Date & JD & Standard \\
\hline 07.05 .2016 & 2457516 & $4 \mathrm{Aql}$ \\
05.06 .2016 & 2457545 & $50 \mathrm{Boo}$ \\
08.06 .2016 & 2457548 & $4 \mathrm{Aql}$ \\
27.07 .2016 & 2457597 & $4 \mathrm{Aql}$ \\
25.10 .2016 & 2457687 & - \\
27.06 .2017 & 2457932 & $4 \mathrm{Aql}$ \\
30.06 .2017 & 2457935 & $4 \mathrm{Aql}$ \\
16.10 .2017 & 2458043 & $4 \mathrm{Aql}$ \\
\hline
\end{tabular}

Table 4: Equivalent widths for V1427 Aql and the star effective temperature estimates.

\begin{tabular}{|c|c|c|c|c|c|c|c|c|c|c|c|}
\hline \multirow[t]{2}{*}{ Date } & \multirow[t]{2}{*}{ JD } & \multicolumn{9}{|c|}{$E W, \AA$} & \multirow[t]{2}{*}{$\overline{T_{e f f}, \mathrm{~K}}$} \\
\hline & & $\mathrm{H} \alpha$ & FeII(6516) & $\operatorname{BaII}(6497)$ & BaII(5854) & $\mathrm{OI}(7774)$ & $\mathrm{P} 12$ & $\mathrm{P} 14$ & $\mathrm{P} 17$ & $\mathrm{CaT}$ & \\
\hline 12.09 .1994 & 2449608 & - & - & - & - & 2.9 & 2.0 & 2.4 & 1.5 & 12.5 & 6835 \\
\hline 30.08 .1996 & 2450326 & - & - & - & - & 3.0 & 1.8 & 2.8 & 1.3 & 13.6 & 6720 \\
\hline 03.08.1998 & 2451029 & 1.90 & 0.70 & 1.40 & 0.60 & 2.9 & - & - & - & - & - \\
\hline 07.08.1999 & 2451398 & 1.97 & 0.86 & 1.11 & 0.29 & 2.9 & 2.0 & 2.5 & 1.6 & 13.6 & 6766 \\
\hline 20.09.1999 & 2451442 & 1.79 & 0.81 & 0.93 & 0.40 & - & 2.3 & 2.3 & 1.7 & 12.8 & 6893 \\
\hline 25.07 .2000 & 2451751 & 1.10 & 0.68 & 1.20 & 0.46 & - & - & - & - & - & - \\
\hline 11.10.2001 & 2452194 & 1.10 & 0.63 & 1.50 & 0.33 & - & - & - & - & - & - \\
\hline 02.07 .2003 & 2452823 & 0.70 & 0.82 & 1.50 & - & - & - & - & - & - & - \\
\hline 09.08 .2005 & 2453592 & 1.40 & 0.78 & 1.40 & - & - & - & - & - & - & - \\
\hline 30.08 .2006 & 2453978 & 1.40 & 0.78 & 1.50 & 0.31 & - & - & - & - & - & - \\
\hline 06.07 .2008 & 2454654 & 1.70 & 0.84 & 1.30 & 0.24 & 2.6 & 2.0 & 2.6 & 1.6 & 13.9 & 6759 \\
\hline 01.08 .2008 & 2454680 & 1.70 & 0.69 & 1.21 & 0.18 & - & - & - & - & - & - \\
\hline 07.05 .2016 & 2457516 & 1.77 & 0.48 & 1.68 & 0.74 & 2.2 & 1.2 & 1.8 & 1.2 & 19.3 & 5850 \\
\hline 05.06 .2016 & 2457545 & 1.88 & 0.79 & 1.93 & 0.60 & 2.3 & 1.8 & 2.0 & 1.2 & 18.5 & 6124 \\
\hline 08.06.2016 & 2457548 & 1.80 & 0.62 & 1.76 & 0.84 & 2.4 & 1.4 & 1.9 & 1.1 & 19.2 & 5922 \\
\hline 27.07 .2016 & 2457597 & 1.58 & 0.63 & 1.82 & 0.80 & 2.2 & 1.7 & 2.0 & 1.1 & 18.3 & 6080 \\
\hline 25.10 .2016 & 2457687 & 1.67 & 0.57 & 1.67 & 0.58 & 2.4 & 1.9 & 2.0 & 1.0 & 18.7 & 6100 \\
\hline 27.06 .2017 & 2457932 & 1.90 & 0.91 & 2.15 & 0.56 & 2.6 & 1.8 & 1.5 & 1.1 & 19.2 & 5920 \\
\hline 30.06 .2017 & 2457935 & 1.79 & 0.90 & 2.08 & 0.54 & 2.5 & 1.8 & 1.5 & 1.1 & 18.4 & 5924 \\
\hline 16.10 .2017 & 2458043 & 1.58 & 0.83 & 2.08 & 0.77 & 2.5 & 1.7 & 2.0 & 0.9 & 19.2 & 5975 \\
\hline
\end{tabular}

